\title{
Dynamical Behavior of a Stochastic Delayed One-Predator and Two-Mutualistic-Prey Model with Markovian Switching and Different Functional Responses
}

\author{
Rensheng He, Zuoliang Xiong, and Desheng Hong \\ Department of Mathematics, Nanchang University, Nanchang, Jiangxi 330031, China \\ Correspondence should be addressed to Zuoliang Xiong; xiong1601@163.com
}

Received 5 March 2016; Revised 14 May 2016; Accepted 21 June 2016

Academic Editor: Seenith Sivasundaram

Copyright (C) 2016 Rensheng He et al. This is an open access article distributed under the Creative Commons Attribution License, which permits unrestricted use, distribution, and reproduction in any medium, provided the original work is properly cited.

We propose a stochastic delayed one-predator and two-mutualistic-prey model perturbed by white noise and telegraph noise. By the $M$-matrix analysis and Lyapunov functions, sufficient conditions of stochastic permanence and extinction are established, respectively. These conditions are all dependent on the subsystems' parameters and the stationary probability distribution of the Markov chain. We also investigate another asymptotic property and finally give two examples and numerical simulations to illustrate main results.

\section{Introduction}

Mutualism plays a key part in ecology, and researchers have proposed many mathematical models to describe the mutualistic interaction [1-6]. In particular, motivated by Holling type II functional response [7], Wright [5] established the Holling type II mutualistic model:

$$
\begin{aligned}
& \dot{x}(t)=x\left(a_{1}+\frac{b_{1} y}{k_{1}+y}-c_{1} x\right), \\
& \dot{y}(t)=y\left(a_{2}+\frac{b_{2} x}{k_{2}+x}-c_{2} y\right) .
\end{aligned}
$$

For the biological meaning of parameters in the above model, we refer to $[5,6,8]$.

Besides, the predator-prey interaction is extremely common in the natural world, and many researchers have paid attention to the predator-prey model. Predator-prey models with Holling types I, II, III, and IV responses were investigated in [9-12]. The Beddington-DeAngelis, CrowleyMartin, and ratio-dependent functional responses were also further considered in [13-15]. But limited work is available on predator-prey model with mutualism. By considering the coexistence of antagonism, mutualism, and competition, Mougi and Kondoh [16] showed that interaction-type diversity generally enhanced stability of complex communities. Motivated by the above ideas, we consider the following onepredator and two-mutualistic-prey model with Holling type II and Beddington-DeAngelis responses:

$$
\begin{aligned}
& \dot{x}(t)=x\left(a_{1}+\frac{b_{1} y}{k_{1}+y}-c_{1} x-\frac{e_{1} z}{1+f_{1} x}\right), \\
& \dot{y}(t)=y\left(a_{2}+\frac{b_{2} x}{k_{2}+x}-c_{2} y-\frac{e_{2} z}{1+f_{2} y+f_{3} z}\right), \\
& \dot{z}(t)=z\left(a_{3}+\frac{e_{3} x}{1+f_{1} x}+\frac{e_{4} y}{1+f_{2} y+f_{3} z}-c_{3} z\right),
\end{aligned}
$$

where species $x, y$ are two mutualistic preys and $z$ is the predator. Furthermore, it is more realistic and reasonable that the future state of population dynamics is determined by not only the present states but also the past $[17,18]$. Up to now, there have been many works considering the effect of time delay $[13,14,19,20]$. Then, taking time delay on mutualistic 
interaction and predation into account, model (2) can be modified as the following model:

$$
\begin{aligned}
\dot{x}(t) & =x\left(a_{1}+\frac{b_{1} y\left(t-\tau_{1}\right)}{k_{1}+y\left(t-\tau_{1}\right)}-c_{1} x-\frac{e_{1} z}{1+f_{1} x}\right), \\
\dot{y}(t) & =y\left(a_{2}+\frac{b_{2} x\left(t-\tau_{2}\right)}{k_{2}+x\left(t-\tau_{2}\right)}-c_{2} y\right. \\
- & \left.\frac{e_{2} z}{1+f_{2} y+f_{3} z}\right) \\
\dot{z}(t) & =z\left(a_{3}+\frac{e_{3} x\left(t-\tau_{3}\right)}{1+f_{1} x\left(t-\tau_{3}\right)}\right. \\
+ & \left.\frac{e_{4} y\left(t-\tau_{4}\right)}{1+f_{2} y\left(t-\tau_{4}\right)+f_{3} z\left(t-\tau_{4}\right)}-c_{3} z\right),
\end{aligned}
$$

where $\tau_{i}>0(i=1,2,3,4)$ denotes the time delay, and we drop $t$ from $x(t), y(t)$, and $z(t)$ and do that throughout this paper.

However, it is not enough to only consider certain factors. The biological system is more or less affected by stochastic fluctuations. One of these general fluctuations is white noise. Recently, many authors have studied lots of stochastic models with white noise, for example, $[12,19,21]$. They mostly put the effect of white noise on the intrinsic birth rate and death rate. In this paper, we assume that white noise affects the intrinsic birth rate and intraspecific competition rate; that is,

$$
\begin{aligned}
& a_{i} \longrightarrow a_{i}+\sigma_{2 i-1} \dot{B}_{2 i-1}(t), \\
& -c_{i} \longrightarrow-c_{i}+\sigma_{2 i} \dot{B}_{2 i}(t), \\
& \quad i=1,2,3,
\end{aligned}
$$

where $B_{j}(t), j=1, \ldots, 6$, denoting white noise, are independent standard Brownian motions and $\sigma_{j}^{2}(j=1, \ldots, 6)$ denotes the intensity of white noise.

Besides white noise, the biological system is inevitably affected by another environment noise, that is, telegraph noise. This noise can be represented by switching among two or more regimes of environment, which are distinguished by factors such as rain falls and nutrition [22, 23]. Suppose $\{r(t), t \geq 0\}$ is a Markov chain controlling the switching among regimes and taking values in a finite state space $\mathbb{S}=$ $\{1,2, \ldots, N\}$. Then, taking white noise and telegraph noise into consideration and on the basis of model (3), we finally developed the following stochastic delayed one-predator and two-mutualistic-prey model with Markovian switching and different functional responses:

$$
\begin{aligned}
& \mathrm{d} x(t)=x\left(a_{1}(r(t))+\frac{b_{1}(r(t)) y\left(t-\tau_{1}\right)}{k_{1}(r(t))+y\left(t-\tau_{1}\right)}\right. \\
& \left.-c_{1}(r(t)) x-\frac{e_{1}(r(t)) z}{1+f_{1}(r(t)) x}\right) \mathrm{d} t+\sigma_{1}(r(t)) \\
& \cdot x \mathrm{~d} B_{1}(t)+\sigma_{2}(r(t)) x^{2} \mathrm{~d} B_{2}(t)
\end{aligned}
$$

$$
\begin{aligned}
& \mathrm{d} y(t)=y\left(a_{2}(r(t))+\frac{b_{2}(r(t)) x\left(t-\tau_{2}\right)}{k_{2}(r(t))+x\left(t-\tau_{2}\right)}\right. \\
& \left.-c_{2}(r(t)) y-\frac{e_{2}(r(t)) z}{1+f_{2}(r(t)) y+f_{3}(r(t)) z}\right) \mathrm{d} t \\
& +\sigma_{3}(r(t)) y \mathrm{~d} B_{3}(t)+\sigma_{4}(r(t)) y^{2} \mathrm{~d} B_{4}(t) \\
& \mathrm{d} z(t)=z\left(a_{3}(r(t))+\frac{e_{3}(r(t)) x\left(t-\tau_{3}\right)}{1+f_{1}(r(t)) x\left(t-\tau_{3}\right)}\right. \\
& +\frac{e_{4}(r(t)) y\left(t-\tau_{4}\right)}{1+f_{2}(r(t)) y\left(t-\tau_{4}\right)+f_{3}(r(t)) z\left(t-\tau_{4}\right)} \\
& \left.-c_{3}(r(t)) z\right) \mathrm{d} t+\sigma_{5}(r(t)) z \mathrm{~d} B_{5}(t)+\sigma_{6}(r(t)) \\
& \cdot z^{2} \mathrm{~d} B_{6}(t),
\end{aligned}
$$

with the initial data

$$
\begin{aligned}
& x(\theta)=\phi_{1}(\theta)>0, \\
& y(\theta)=\phi_{2}(\theta)>0, \\
& z(\theta)=\phi_{3}(\theta)>0,
\end{aligned}
$$

$$
\theta \in[-\tau, 0]
$$

where $r(0)=r_{0} \in \mathbb{S}, \tau=\max _{1 \leq i \leq 4} \tau_{i}$, and all of the parameters are positive. In regime $i(i \in \mathbb{S}$ ), system (5) obeys

$$
\begin{aligned}
& \mathrm{d} x(t)=x\left(a_{1}(i)+\frac{b_{1}(i) y\left(t-\tau_{1}\right)}{k_{1}(i)+y\left(t-\tau_{1}\right)}-c_{1}(i) x\right. \\
& \left.-\frac{e_{1}(i) z}{1+f_{1}(i) x}\right) \mathrm{d} t+\sigma_{1}(i) x \mathrm{~d} B_{1}(t)+\sigma_{2}(i) \\
& \cdot x^{2} \mathrm{~d} B_{2}(t), \\
& \mathrm{d} y(t)=y\left(a_{2}(i)+\frac{b_{2}(i) x\left(t-\tau_{2}\right)}{k_{2}(i)+x\left(t-\tau_{2}\right)}-c_{2}(i) y\right. \\
& -\frac{e_{2}(i) z}{1+f_{2}(i) y+f_{3}(i) z} \mathrm{~d} t+\sigma_{3}(i) y \mathrm{~d} B_{3}(t) \\
& +\sigma_{4}(i) y^{2} \mathrm{~d} B_{4}(t), \\
& \mathrm{d} z(t)=z\left(a_{3}(i)+\frac{e_{3}(i) x\left(t-\tau_{3}\right)}{1+f_{1}(i) x\left(t-\tau_{3}\right)}\right. \\
& +\frac{e_{4}(i) y\left(t-\tau_{4}\right)}{1+f_{2}(i) y\left(t-\tau_{4}\right)+f_{3}(i) z\left(t-\tau_{4}\right)} \\
& \left.-c_{3}(i) z\right) \mathrm{d} t+\sigma_{5}(i) z \mathrm{~d} B_{5}(t)+\sigma_{6}(i) z^{2} \mathrm{~d} B_{6}(t)
\end{aligned}
$$

Therefore, (7) is regarded as the subsystem of system (5). In this paper, our main aim is to reveal how two kinds of 
environment noise, that is, white noise and telegraph noise, affect permanence and extinction of system (5).

The stochastic differential equations controlled by a continuous Markov chain have been applied to the population models with telegraph noise. Li et al. [24] investigated the logistic population system without intraspecific competition incorporating white and telegraph noise and mainly researched stochastic permanence and extinction. A two-dimensional stochastic predator-prey model with Markovian switching was developed by Ouyang and Li [15], and they explored permanence and asymptotical behavior. However, for the stochastic predator-prey model with Markovian switching, most of previous works focused on twodimensional systems. And to the best of our knowledge, there is no work about 3-dimensional stochastic delayed predatorprey models with Markovian switching, two mutualistic preys, and different functional responses till now.

We arrange the rest of this paper as follows. In Section 2, we prepare some notations and consider the existence and uniqueness of the solution of system (5). By the $M$-matrix analysis and Lyapunov functions, we study stochastically ultimate boundedness and stochastic permanence, and the sufficient condition of stochastic permanence is given in Section 3. Section 4 gives the sample Lyapunov exponent and hence shows the sufficient condition of extinction. We study another asymptotic property in Section 5. In Section 6, we give two examples and make numerical simulations to illustrate main results and reveal the dynamical behavior. In Section 7, we give conclusions and the future direction.

\section{Preliminaries}

Throughout this paper, let $(\Omega, \mathscr{F}, \mathbb{P})$ be a complete probability space with the filtration $\left\{\mathscr{F}_{t}\right\}_{t \geq 0}$ satisfying the usual conditions (i.e., it is increasing and right continuous while $\mathscr{F}_{0}$ contains all $P$-null sets). Denote by $\mathbb{R}_{+}^{n}$ the positive cone in $\mathbb{R}^{n}$. Let $C\left([-\tau, 0] ; \mathbb{R}_{+}^{3}\right)$ denote the space of continuous function $\phi:[-\tau, 0] \rightarrow \mathbb{R}_{+}^{3}$ with the norm $\|\phi\|=\sup _{-\tau \leq \theta \leq 0}|\phi(\theta)|$. Denote by $X(t)=(x(t), y(t), z(t))$ a solution of system (5) and its norm is defined by $|X(t)|=\sqrt{x^{2}(t)+y^{2}(t)+z^{2}(t)}$. Let $x_{\tau_{i}}, y_{\tau_{i}}, z_{\tau_{i}}(i=1,2,3,4)$ denote $x\left(t-\tau_{i}\right), y\left(t-\tau_{i}\right), z\left(t-\tau_{i}\right)$, respectively. Let the initial data of system (5) be

$$
\begin{array}{r}
\{(x(\theta), y(\theta), z(\theta)):-\tau \leq \theta \leq 0\} \in C\left([-\tau, 0] ; \mathbb{R}_{+}^{3}\right) \\
r(0)=r_{0} \in \mathbb{S} .
\end{array}
$$

Assume that $r(t)$ is a right-continuous Markov chain taking values in the finite state space $\mathbb{S}$ with the generator $\Gamma=\left(\gamma_{m n}\right)_{N \times N}$ defined by

$$
\begin{aligned}
& \mathbb{P}\{r(t+\delta)=n \mid r(t)=m\} \\
& = \begin{cases}\gamma_{m n} \delta+o(\delta), & \text { if } n \neq m, \\
1+\gamma_{m n} \delta+o(\delta), & \text { if } n=m,\end{cases}
\end{aligned}
$$

where $\delta>0$. Here $\gamma_{m n}$ is the transition rate from $m$ to $n$ and $\gamma_{m n} \geq 0$ if $n \neq m$, while

$$
\gamma_{m m}=-\sum_{n=1, n \neq m}^{N} \gamma_{m n}, \quad \forall m \in \mathbb{S} .
$$

We also assume that the Markov chain $r(t)$ is independent of the Brownian motion $B_{i}(t)(i=1, \ldots, 6)$ and is irreducible (e.g., [18]), which indicates that the overall system (5) can switch from any regime to any another regime. Under this assumption, the Markov chain $r(t)$ has a unique stationary probability distribution $\pi=\left(\pi_{1}, \pi_{2}, \ldots, \pi_{N}\right) \in \mathbb{R}^{1 \times N}$, depending on the equation

$$
\pi \Gamma=0
$$

subject to

$$
\sum_{i=1}^{N} \pi_{i}=1, \quad \pi_{i}>0, \forall i \in \mathbb{S} .
$$

For convenience and simplicity, we give the following notations:

$$
\begin{aligned}
& B(i)=\min \left\{a_{1}(i), a_{2}(i), a_{3}(i)-\frac{e_{1}(i)}{f_{1}(i)}-\frac{e_{2}(i)}{f_{2}(i)}\right\}, \\
& C(i)=\max \left\{\sigma_{1}^{2}(i), \sigma_{3}^{2}(i), \sigma_{5}^{2}(i)\right\}, \\
& D(i)=\max \left\{c_{1}(i), c_{2}(i), c_{3}(i)\right\}, \\
& E(i)=\max \left\{\sigma_{2}^{2}(i), \sigma_{4}^{2}(i), \sigma_{6}^{2}(i)\right\}, \\
& \widehat{g}=\min _{i \in \mathbb{S}} g_{i}, \\
& F_{1}(x, y, z, r(t)) \\
& =a_{1}(r(t))+\frac{b_{1}(r(t)) y_{\tau_{1}}}{k_{1}(r(t))+y_{\tau_{1}}}-c_{1}(r(t)) x \\
& -\frac{e_{1}(r(t)) z}{1+f_{1}(r(t)) x} \\
& F_{2}(x, y, z, r(t)) \\
& =a_{2}(r(t))+\frac{b_{2}(r(t)) x_{\tau_{2}}}{k_{2}(r(t))+x_{\tau_{2}}}-c_{2}(r(t)) y \\
& -\frac{e_{2}(r(t)) z}{1+f_{2}(r(t)) y+f_{3}(r(t)) z}, \\
& F_{3}(x, y, z, r(t)) \\
& =a_{3}(r(t))+\frac{e_{3}(r(t)) x_{\tau_{3}}}{1+f_{1}(r(t)) x_{\tau_{3}}} \\
& +\frac{e_{4}(r(t)) y_{\tau_{4}}}{1+f_{2}(r(t)) y_{\tau_{4}}+f_{3}(r(t)) z_{\tau_{4}}} \\
& -c_{3}(r(t)) z(t),
\end{aligned}
$$




$$
\begin{aligned}
M_{1}(x, y, z, r(t)) & \\
= & \sigma_{1}(r(t)) x \mathrm{~d} B_{1}(t)+\sigma_{2}(r(t)) x^{2} \mathrm{~d} B_{2}(t) \\
& +\sigma_{3}(r(t)) y \mathrm{~d} B_{3}(t)+\sigma_{4}(r(t)) y^{2} \mathrm{~d} B_{4}(t) \\
& \quad+\sigma_{5}(r(t)) z \mathrm{~d} B_{5}(t)+\sigma_{6}(r(t)) z^{2} \mathrm{~d} B_{6}(t), \\
N(x, y, z, r(t)) & \\
= & \sigma_{1}^{2}(r(t)) x^{2}+\sigma_{2}^{2}(r(t)) x^{4}+\sigma_{3}^{2}(r(t)) y^{2} \\
& +\sigma_{4}^{2}(r(t)) y^{4}+\sigma_{5}^{2}(r(t)) z^{2}+\sigma_{6}^{2}(r(t)) z^{4} .
\end{aligned}
$$

In order to study the dynamic behavior, we must firstly guarantee that there exists a unique, positive, and global solution.

Theorem 1. For any initial data (8), there is a unique positive solution of system (5) on $t \geq-\tau$, which remains in $\mathbb{R}_{+}^{3} \times \mathbb{S}$ with probability 1 .

Proof. Define a function $V: \mathbb{R}_{+}^{3} \times \mathbb{S} \rightarrow \mathbb{R}_{+}$by

$$
\begin{aligned}
V(x, y, z, i)= & (\sqrt{x}-1-0.5 \log x) \\
& +(\sqrt{y}-1-0.5 \log y) \\
& +(\sqrt{z}-1-0.5 \log z) .
\end{aligned}
$$

This proof is standard, so please refer to $[13,25]$, and thus we omit it.

\section{Stochastic Permanence}

In this section, we will consider stochastic permanence and firstly study stochastically ultimate boundedness.

Definition 2 (e.g., see [24]). System (5) is said to be stochastically permanent if for any $\epsilon \in(0,1)$ there are two positive constants $\chi=\chi(\epsilon)$ and $\delta=\delta(\epsilon)$ such that for any initial data (8) the solution $X(t)$ of system (5) satisfies

$$
\begin{aligned}
& \liminf _{t \rightarrow \infty} \mathbb{P}\{|X(t)| \geq \chi\} \geq 1-\epsilon, \\
& \liminf _{t \rightarrow \infty} \mathbb{P}\{|X(t)| \leq \delta\} \geq 1-\epsilon .
\end{aligned}
$$

Lemma 3. Let $0<p<1$. Then there is a constant $H_{1}=$ $H_{1}(p)>0$, which is independent of the initial data (8), such that the solution $X(t)$ of system (5) satisfies

$$
\limsup _{t \rightarrow \infty} \mathbf{E}|X(t)|^{p} \leq H_{1} .
$$

Proof. Define a function

$$
V(x, y, z, i)=x^{p}+y^{p}+z^{p}
$$

By means of the generalized Itô formula (e.g., see [18]), we get

$$
\begin{aligned}
& \mathscr{L} V(x, y, z, i)=p x^{p}\left(F_{1}(x, y, z, i)-\frac{1-p}{2} \sigma_{1}^{2}(i)\right. \\
& \left.-\frac{1-p}{2} \sigma_{2}^{2}(i) x^{2}\right)+p y^{p}\left(F_{2}(x, y, z, i)\right. \\
& \left.-\frac{1-p}{2} \sigma_{3}^{2}(i)-\frac{1-p}{2} \sigma_{4}^{2}(i) y^{2}\right) \\
& +p z^{p}\left(F_{3}(x, y, z, i)-\frac{1-p}{2} \sigma_{5}^{2}(i)\right. \\
& \left.-\frac{1-p}{2} \sigma_{6}^{2}(i) z^{2}\right) \leq p x^{p}\left(a_{1}(i)+b_{1}(i)+\frac{1}{p}\right. \\
& \left.-\frac{1-p}{2} \sigma_{2}^{2}(i) x^{2}\right)+p y^{p}\left(a_{2}(i)+b_{2}(i)+\frac{1}{p}\right. \\
& \left.-\frac{1-p}{2} \sigma_{4}^{2}(i) y^{2}\right)+p z^{p}\left(a_{3}(i)+\frac{e_{3}(i)}{f_{1}(i)}+\frac{e_{4}(i)}{f_{2}(i)}\right. \\
& \left.+\frac{1}{p}-\frac{1-p}{2} \sigma_{6}^{2}(i) z^{2}\right)-V(x, y, z, i)
\end{aligned}
$$

Note that $0<p<1, \mathscr{L} V(x, y, z, i) \leq H_{11}-V(x, y, z, i)$, where $H_{11}$ is a positive constant. Then applying the generalized Itô formula to $e^{t} V(x, y, z, i)$, we get

$$
\begin{aligned}
\mathscr{L} & \left(e^{t} V(x, y, z, i)\right) \\
& =e^{t}[V(x, y, z, i)+\mathscr{L} V(x, y, z, i)] \leq H_{11} e^{t} .
\end{aligned}
$$

Integrating both sides of $\mathrm{d}\left(e^{t} V(x, y, z, i)\right)$ from 0 to $t$, taking the expectation, and taking the limit superior, we obtain $\lim \sup _{t \rightarrow \infty} \mathbf{E} V(x(t), y(t), z(t), i) \leq H_{11}$. Note that $|X(t)|^{p}=$ $|(x, y, z)|^{p} \leq 3^{p / 2} \max \left\{x^{p}, y^{p}, z^{p}\right\} \leq 3^{p / 2} V(x, y, z)$, and thus we have

$$
\limsup _{t \rightarrow \infty} \mathbf{E}|X(t)|^{p} \leq 3^{p / 2} H_{11}:=H_{1}(p) .
$$

Theorem 4. System (5) is stochastically ultimately bounded.

Proof. Let $p \in(0,1)$. By the definition of stochastically ultimate boundedness (e.g., see [24]), the conclusion follows from Lemma 3 and Chebyshev's inequality.

Next we will investigate stochastic permanence. Based on the above conclusion, we only need to prove another inequality about stochastic permanence. And one of the main methods in this section is the $M$-matrix analysis which was introduced by [18] and used in [15, 24].

Now we give notations, the classical result, and some assumptions. Let $A$ be a vector or matrix. Denote by $A \gg 0$ that all elements of $A$ are positive. Set

$$
Z^{N \times N}=\left\{A=\left(a_{i j}\right)_{N \times N}: a_{i j} \leq 0, i \neq j\right\} .
$$


Lemma 5 (e.g., see [18]). If $A \in Z^{N \times N}$, then the following statements are equivalent:

(i) $A$ is a nonsingular M-matrix.

(ii) A is semipositive; that is, there exists $x \gg 0$ in $\mathbb{R}^{N}$ such that $A x \gg 0$.

Assumption (A1). For some $n \in \mathbb{S}, \gamma_{i n}>0, \forall i \neq n$.

Assumption (A2). $\sum_{i=1}^{N} \pi_{i} q(i)>0$, where $q(i)=B(i)-$ $(1 / 2) C(i)$

Assumption (A3). For some $i \in \mathbb{S}, q(i)=B(i)-(1 / 2) C(i)>0$.

The proof of stochastic permanence is rather long and technical. To make it more understandable, we divide the proof into several lemmas.

Lemma 6. Assumptions (A1) and (A2) imply that there exists a constant $\alpha>0$ such that the matrix

$$
G(\alpha)=\operatorname{diag}\left\{\beta_{1}(\alpha), \beta_{2}(\alpha), \ldots, \beta_{N}(\alpha)\right\}-\Gamma
$$

is a nonsingular M-matrix, where

$$
\beta_{i}(\alpha)=\alpha q(i)-\frac{1}{2} \alpha^{2} C(i), \quad i \in \mathbb{S} .
$$

Proof. This proof is standard, so please refer to $[15,24]$, and thus we omit it.

Lemma 7. If there is a constant $\alpha>0$ such that $G(\alpha)$ is a nonsingular $M$-matrix, then the solution $X(t)$ of system (5) with any initial data (8) satisfies

$$
\limsup _{t \rightarrow \infty} \mathbf{E} \frac{1}{|X(t)|^{\alpha}} \leq H_{2},
$$

where $\mathrm{H}_{2}=\mathrm{H}_{2}(\alpha)$ is a positive constant.

Proof. Define $V(x, y, z, i)=x+y+z$. By the generalized Itô formula, we have

$$
\mathrm{d} V(x, y, z, i)=\mathscr{L} V(x, y, z, i) \mathrm{d} t+M_{1}(x, y, z, i),
$$

where

$$
\begin{aligned}
\mathscr{L} V(x, y, z, i)= & x F_{1}(x, y, z, i)+y F_{2}(x, y, z, i) \\
& +z F_{3}(x, y, z, i) .
\end{aligned}
$$

Define again $U(x, y, z, i)=1 / V(x, y, z, i)$. By the generalized Itô formula, we get

$$
\begin{aligned}
\mathrm{d} U & (x, y, z, i) \\
= & {\left[-U^{2} \mathscr{L} V(x, y, z, i)+U^{3} N(x, y, z, i)\right] \mathrm{d} t } \\
& -U^{2} M_{1}(x, y, z, i) .
\end{aligned}
$$

For given $\alpha>0$, by Lemma 5 , there exists a vector $\vec{\eta}=$ $\left(\eta_{1}, \ldots, \eta_{N}\right)^{T} \gg 0$ such that $G(\alpha) \vec{\eta} \gg 0$; that is,

$$
\beta_{i}(\alpha) \eta_{i}-\sum_{j=1}^{N} \gamma_{i j} \eta_{j}>0, \quad i \in \mathbb{S} .
$$

Define the third function $\bar{V}(x, y, z, i)=\eta_{i} U^{\alpha}(x, y, z, i)$. By the generalized Itô formula, we have

$$
\begin{gathered}
\mathscr{L} \bar{V}(x, y, z, i)=U^{\alpha-2}\left\{\eta _ { i } \left[-\alpha U^{3} \mathscr{L} V(x, y, z, i)\right.\right. \\
\left.\left.+\frac{\alpha(\alpha+1)}{2} U^{4} N(x, y, z, i)\right]+U^{2} \sum_{j=1}^{N} \gamma_{i j} \eta_{j}\right\} .
\end{gathered}
$$

Computing

$$
\begin{aligned}
& -U^{3} \mathscr{L} V(x, y, z, i)=-U^{3}\left(x F_{3}(x, y, z, i)\right. \\
& \left.+y F_{2}(x, y, z, i)+z F_{3}(x, y, z, i)\right) \\
& \leq-U^{3}\left[x\left(a_{1}(i)-c_{1}(i) x-\frac{e_{1}(i) z}{f_{1}(i) x}\right)\right. \\
& +y\left(a_{2}(i)-c_{2}(i) y-\frac{e_{2}(i) z}{f_{2}(i) y}\right) \\
& \left.+z\left(a_{3}(i)-c_{3}(i) z\right)\right]=-U^{3}\left\{a_{1}(i) x+a_{2}(i) y\right. \\
& +\left(a_{3}(i)-\frac{e_{1}(i)}{f_{1}(i)}-\frac{e_{2}(i)}{f_{2}(i)}\right) z \\
& \left.\quad-\left[c_{1}(i) x^{2}+c_{2}(i) y^{2}+c_{3}(i) z^{2}\right]\right\} \\
& \quad \leq-\min \left\{a_{1}(i), a_{2}(i), a_{3}(i)-\frac{e_{1}(i)}{f_{1}(i)}-\frac{e_{2}(i)}{f_{2}(i)}\right\} U^{2} \\
& +\max \left\{c_{1}(i), c_{2}(i), c_{3}(i)\right\} U=-B(i) U^{2}+D(i) U, \\
& U^{4} N(x, y, z, i) \leq \max \left\{\sigma_{1}^{2}(i), \sigma_{3}^{2}(i), \sigma_{5}^{2}(i)\right\} U^{2} \\
& +\max \left\{\sigma_{2}^{2}(i), \sigma_{4}^{2}(i), \sigma_{6}^{2}(i)\right\}=C(i) U^{2}+E(i)
\end{aligned}
$$

and substituting (30) into (29), we obtain

$$
\begin{aligned}
& \mathscr{L} \bar{V}(x, y, z, i) \\
& \leq U^{\alpha-2}\left\{-\left[\eta_{i}\left(\alpha B(i)-\frac{\alpha(\alpha+1)}{2} C(i)\right)\right.\right. \\
& \left.\left.\quad-\sum_{j=1}^{N} \gamma_{i j} \eta_{j}\right] U^{2}+\eta_{i} \alpha D(i) U+\frac{\eta_{i} \alpha(\alpha+1)}{2} E(i)\right\} .
\end{aligned}
$$

Under (28), there is a sufficiently small constant $l>0$ such that $G(\alpha) \vec{\eta}-l \vec{\eta} \gg 0$; that is,

$$
\beta_{i}(\alpha) \eta_{i}-\sum_{j=1}^{N} \gamma_{i j} \eta_{j}-\ln \eta_{i}>0, \quad i \in \mathbb{S} .
$$


Applying the generalized Itô formula to $e^{l t} \bar{V}(x, y, z, i)$ and noticing (23), we obtain

$$
\begin{aligned}
& \mathscr{L}\left[e^{l t} \bar{V}(x, y, z, i)\right]=l e^{l t} \bar{V}(x, y, z, i) \\
& +e^{l t} \mathscr{L} \bar{V}(x, y, z, i) \leq e^{l t} U^{\alpha-2}\left\{\frac{\eta_{i} \alpha(\alpha+1)}{2} E(i)\right. \\
& \left.+\eta_{i} \alpha D(i) U-\left[\beta_{i}(\alpha) \eta_{i}-\sum_{j=1}^{N} \gamma_{i j} \eta_{j}-l \eta_{i}\right] U^{2}\right\} .
\end{aligned}
$$

By (32), it is obvious that $\mathscr{L}\left[e^{l t} \bar{V}(x, y, z, i)\right] \leq H_{22} e^{l t}$, where

$$
\begin{aligned}
H_{22} & =\max _{i \in \mathbb{S}}\left\{\operatorname { s u p } _ { U \in \mathbb { R } _ { + } } U ^ { \alpha - 2 } \left\{\frac{\eta_{i} \alpha(\alpha+1)}{2} E(i)\right.\right. \\
& \left.+\eta_{i} \alpha D(i) U-\left[\beta_{i}(\alpha) \eta_{i}-\sum_{j=1}^{N} \gamma_{i j} \eta_{j}-l \eta_{i}\right] U^{2}\right\}, \\
& 1\} .
\end{aligned}
$$

Thus, $\limsup _{t \rightarrow \infty} \mathbf{E} \bar{V}(x(t), y(t), z(t), i) \leq H_{22}$. Noting that $(x+y+z)^{\alpha} \leq 3^{\alpha}|(x, y, z)|^{\alpha}$, we have

$$
\limsup _{t \rightarrow \infty} \mathbf{E} \frac{1}{|X(t)|^{\alpha}} \leq \frac{3^{\alpha}}{\widehat{\eta}} H_{22}:=H_{2} .
$$

Theorem 8. Under Assumptions (A1) and (A2), system (5) is stochastically permanent.

Proof. By Lemma 7, Chebyshev's inequality, and Theorem 4, we can get the desired conclusion.

On the basis of the above theorem, we directly give the following corollary about subsystems permanence.

Corollary 9. Under Assumption (A3), subsystem (7) is stochastically permanent.

\section{Extinction}

In this section, we will discuss the sample Lyapunov exponent of system (5) and hence get the sufficient condition for three species to be extinct.

Theorem 10. For any initial data (8), the solution $X(t)$ of system (5) has the property that

$$
\limsup _{t \rightarrow \infty} \frac{\ln x(t)}{t} \leq \sum_{i \in \mathbb{S}} \pi_{i}\left(a_{1}(i)+b_{1}(i)-\frac{1}{2} \sigma_{1}^{2}(i)\right)
$$

$$
\limsup _{t \rightarrow \infty} \frac{\ln y(t)}{t} \leq \sum_{i \in \mathbb{S}} \pi_{i}\left(a_{2}(i)+b_{2}(i)-\frac{1}{2} \sigma_{3}^{2}(i)\right)
$$

$$
\begin{aligned}
& \limsup _{t \rightarrow \infty} \frac{\ln z(t)}{t} \\
& \quad \leq \sum_{i \in \mathbb{S}} \pi_{i}\left(a_{3}(i)+\frac{e_{3}(i)}{f_{1}(i)}+\frac{e_{4}(i)}{f_{2}(i)}-\frac{1}{2} \sigma_{5}^{2}(i)\right) \text { a.s. }
\end{aligned}
$$

Particularly, if $\sum_{i \in \mathbb{S}} \pi_{i}\left(a_{1}(i)+b_{1}(i)-(1 / 2) \sigma_{1}^{2}(i)\right)<0$, $\sum_{i \in \mathbb{S}} \pi_{i}\left(a_{2}(i)+b_{2}(i)-(1 / 2) \sigma_{3}^{2}(i)\right)<0$ and $\sum_{i \in \mathbb{S}} \pi_{i}\left(a_{3}(i)+\right.$ $\left.e_{3}(i) / f_{1}(i)+e_{4}(i) / f_{2}(i)-(1 / 2) \sigma_{5}^{2}(i)\right)<0$, then

$$
\begin{aligned}
& \lim _{t \rightarrow \infty} x(t)=0, \\
& \lim _{t \rightarrow \infty} y(t)=0, \\
& \lim _{t \rightarrow \infty} z(t)=0
\end{aligned}
$$

\section{a.s.}

Proof. By the generalized Itô formula, we have

$$
\begin{aligned}
& \mathrm{d} \ln x(t)=\left(F_{1}(x, y, z, r(t))-\frac{1}{2} \sigma_{1}^{2}(r(t))\right. \\
& \left.-\frac{1}{2} \sigma_{2}^{2}(r(t)) x^{2}(t)\right) \mathrm{d} t+\sigma_{1}(r(t)) \mathrm{d} B_{1}(t) \\
& +\sigma_{2}(r(t)) x(t) \mathrm{d} B_{2}(t) \leq\left(a_{1}(r(t))+b_{1}(r(t))\right. \\
& \left.-\frac{1}{2} \sigma_{1}^{2}(r(t))-\frac{1}{2} \sigma_{2}^{2}(r(t)) x^{2}(t)\right) \mathrm{d} t \\
& +\sigma_{1}(r(t)) \mathrm{d} B_{1}(t)+\sigma_{2}(r(t)) x(t) \mathrm{d} B_{2}(t) .
\end{aligned}
$$

Integrating from 0 to $t$ on both sides of the above inequality, we obtain

$$
\begin{aligned}
& \ln \frac{x(t)}{x(0)} \leq \int_{0}^{t}\left(a_{1}(r(s))+b_{1}(r(s))-\frac{1}{2} \sigma_{1}^{2}(r(s))\right. \\
& \left.-\frac{1}{2} \sigma_{2}^{2}(r(s)) x^{2}(s)\right) \mathrm{d} s+\sigma_{1}(r(t)) B_{1}(t) \\
& \quad+M_{2}(t),
\end{aligned}
$$

where

$$
M_{2}(t)=\int_{0}^{t} \sigma_{2}(r(s)) x(s) \mathrm{d} B_{2}(s)
$$

is real-valued continuous local martingale and its quadratic form is defined by

$$
\left\langle M_{2}(t), M_{2}(t)\right\rangle=\int_{0}^{t} \sigma_{2}^{2}(r(s)) x^{2}(s) \mathrm{d} s .
$$

Let $\epsilon \in(0,1)$ be arbitrary. By the exponential martingale inequality (e.g., see [18]), for each $k \geq 1$,

$$
\begin{aligned}
\mathbb{P} & \left\{\sup _{0 \leq t \leq k}\left[M_{2}(t)-\frac{\epsilon}{2}\left\langle M_{2}(t), M_{2}(t)\right\rangle\right]>\frac{2 \ln k}{\epsilon}\right\} \\
& \leq k^{-2} .
\end{aligned}
$$


Noting that the series $\sum_{k=1}^{\infty} k^{-2}$ converges and by the BorelCantelli lemma (e.g., see [18]), there exists $\Omega_{0} \subseteq \Omega$ with $\mathbb{P}\left(\Omega_{0}\right)=1$ such that, for any $\omega \in \Omega_{0}$, there is an integer $k_{0}=k_{0}(\omega)$ such that

$$
M_{2}(t) \leq \frac{\epsilon}{2}\left\langle M_{2}(t), M_{2}(t)\right\rangle+\frac{2 \ln k}{\epsilon},
$$

for all $0 \leq t \leq k$ and $k \geq k_{0}(\omega)$. Substituting (43) into (39) and noting that $\epsilon \in(0,1)$, we get

$$
\begin{aligned}
\ln \frac{x(t)}{x(0)} \leq & \int_{0}^{t}\left(a_{1}(r(s))+b_{1}(r(s))-\frac{1}{2} \sigma_{1}^{2}(r(s))\right) \mathrm{d} s \\
& +\sigma_{1}(r(t)) B_{1}(t)+\frac{2 \ln k}{\epsilon},
\end{aligned}
$$

for all $0 \leq t \leq k$ and $k \geq k_{0}(\omega)$. Then for any $\omega \in \Omega_{0}$, if $k \leq t \leq k+1$ and $k \geq k_{0}(\omega)$, we obtain

$$
\begin{aligned}
& \frac{\ln [x(t) / x(0)]}{t} \\
& \leq \frac{1}{t} \int_{0}^{t}\left(a_{1}(r(s))+b_{1}(r(s))-\frac{1}{2} \sigma_{1}^{2}(r(s))\right) \mathrm{d} s \\
& \quad+\frac{\sigma_{1}(r(t)) B_{1}(t)}{t}+\frac{2 \ln k}{\epsilon k} .
\end{aligned}
$$

Taking the limit superior on both sides of the above inequality and by the strong law of large numbers and the ergodic property of Markov chain (e.g., see [18]), we finally obtain

$$
\limsup _{t \rightarrow \infty} \frac{\ln x(t)}{t} \leq \sum_{i \in \mathbb{S}} \pi_{i}\left(a_{1}(i)+b_{1}(i)-\frac{1}{2} \sigma_{1}^{2}(i)\right)
$$

a.s.

By the above same methods and procedures, we have

$$
\begin{aligned}
& \limsup _{t \rightarrow \infty} \frac{\ln y(t)}{t} \leq \sum_{i \in \mathbb{S}} \pi_{i}\left(a_{2}(i)+b_{2}(i)-\frac{1}{2} \sigma_{3}^{2}(i)\right) \\
& \limsup _{t \rightarrow \infty} \frac{\ln z(t)}{t} \\
& \leq \sum_{i \in \mathbb{S}} \pi_{i}\left(a_{3}(i)+\frac{e_{3}(i)}{f_{1}(i)}+\frac{e_{4}(i)}{f_{2}(i)}-\frac{1}{2} \sigma_{5}^{2}(i)\right) \text { a.s. }
\end{aligned}
$$

The proof is completed.

On the basis of the above theorem, we directly give the following corollary about subsystem's extinction.

Corollary 11. For subsystem (7), if the solution $(x(t), y(t)$, $z(t))$ satisfies $a_{1}(i)+b_{1}(i)-(1 / 2) \sigma_{1}^{2}(i)<0, a_{2}(i)+b_{2}(i)-$
$(1 / 2) \sigma_{3}^{2}(i)<0$, and $a_{3}(i)+e_{3}(i) / f_{1}(i)+e_{4}(i) / f_{2}(i)-$ $(1 / 2) \sigma_{5}^{2}(i)<0$, then

$$
\begin{aligned}
& \lim _{t \rightarrow \infty} x(t)=0, \\
& \lim _{t \rightarrow \infty} y(t)=0, \\
& \lim _{t \rightarrow \infty} z(t)=0
\end{aligned}
$$

a.s.

\section{Asymptotic Property}

In this section, we will consider another asymptotic property of system (5).

Theorem 12. For any initial data (8), the solution $X(t)$ of system (5) has the property that

$$
\limsup _{t \rightarrow \infty} \frac{\ln |X(t)|}{\ln t} \leq 1 \quad \text { a.s. }
$$

Proof. Define

$$
V=V(X(t))=x(t)+y(t)+z(t) .
$$

Let $\lambda>0$ be arbitrary. Applying the generalized Itô formula to $e^{\lambda t} \ln V(X(t))$, we get

$$
\begin{aligned}
e^{\lambda t} & \ln V(X(t))=\ln V(X(0))+M_{3}(t) \\
+ & \int_{0}^{t} e^{\lambda s}\left[\lambda \ln V(X(s))+\frac{x}{V} F_{1}(x, y, z, r(s))\right. \\
+ & \frac{y}{V} F_{2}(x, y, z, r(s))+\frac{z}{V} F_{3}(x, y, z, r(s)) \\
- & \frac{\sigma_{1}^{2}(r(s)) x^{2}+\sigma_{2}^{2}(r(s)) x^{4}}{2 V^{2}} \\
- & \frac{\sigma_{3}^{2}(r(s)) y^{2}+\sigma_{4}^{2}(r(s)) y^{4}}{2 V^{2}} \\
- & \left.\frac{\sigma_{5}^{2}(r(s)) z^{2}+\sigma_{6}^{2}(r(s)) z^{4}}{2 V^{2}}\right] \mathrm{d} s,
\end{aligned}
$$

where $M_{1}(x, y, z, r(t))$ and $N(x, y, z, r(t))$ are defined in Section 2 .

$$
M_{3}(t)=\int_{0}^{t} \frac{e^{\lambda s}}{V} M_{1}(x(s), y(s), z(s), r(s))
$$

is real-valued continuous local martingale and its quadratic form is defined by

$$
\begin{aligned}
& \left\langle M_{3}(t), M_{3}(t)\right\rangle \\
& \quad=\int_{0}^{t} \frac{e^{2 \lambda s}}{V^{2}} N(x(s), y(s), z(s), r(s)) \mathrm{d} s .
\end{aligned}
$$


Let $\epsilon \in(0,1)$ and $\eta>1$ be arbitrary. By the exponential martingale inequality, for each $k \geq 1$,

$$
\begin{gathered}
\mathbb{P}\left\{\sup _{0 \leq t \leq k}\left[M_{3}(t)-\frac{\epsilon}{2} e^{-\lambda k}\left\langle M_{3}(t), M_{3}(t)\right\rangle\right]\right. \\
\left.>\frac{\eta e^{\lambda k}}{\epsilon} \ln k\right\} \leq k^{-\eta} .
\end{gathered}
$$

Noting that the series $\sum_{k=1}^{\infty} k^{-\eta}$ converges and by the BorelCantelli lemma, there exists $\Omega_{0} \subseteq \Omega$ with $\mathbb{P}\left(\Omega_{0}\right)=1$ such that, for any $\omega \in \Omega_{0}$, there is an integer $k_{0}=k_{0}(\omega)$ such that

$$
M_{3}(t) \leq \frac{\epsilon}{2} e^{-\lambda k}\left\langle M_{3}(t), M_{3}(t)\right\rangle+\frac{\eta e^{\lambda k}}{\epsilon} \ln k,
$$

for all $0 \leq t \leq k$ and $k \geq k_{0}(\omega)$. Note that

$$
\begin{aligned}
\frac{x}{V} F_{1}(x, y, z, r(t)) & \leq a_{1}(r(t))+b_{1}(r(t)), \\
\frac{y}{V} F_{2}(x, y, z, r(t)) & \leq a_{2}(r(t))+b_{2}(r(t)), \\
\frac{z}{V} F_{3}(x, y, z, r(t)) \leq & a_{3}(r(t))+\frac{e_{3}(r(t))}{f_{1}(r(t))} \\
& +\frac{e_{4}(r(t))}{f_{2}(r(t))},
\end{aligned}
$$

and choose three constants $m_{1}, m_{2}, m_{3} \in(0,1)$ such that

$$
\begin{aligned}
& \frac{\sigma_{2}^{2}(r(t)) x^{4}}{2 V^{2}} \leq \frac{\sigma_{2}^{2}(r(t)) m_{1}}{2} x^{2}, \\
& \frac{\sigma_{4}^{2}(r(t)) y^{4}}{2 V^{2}} \leq \frac{\sigma_{2}^{2}(r(t)) m_{2}}{2} y^{2}, \\
& \frac{\sigma_{6}^{2}(r(t)) z^{4}}{2 V^{2}} \leq \frac{\sigma_{6}^{2}(r(t)) m_{3}}{2} z^{2} .
\end{aligned}
$$

Substituting (55)-(57) into (51) and noting that $\epsilon \in(0,1)$, we obtain

$$
\begin{aligned}
e^{\lambda t} & \ln V(X(t)) \leq \ln V(X(0))+\frac{\eta e^{\lambda k}}{\epsilon} \ln k \\
+ & \int_{0}^{t} e^{\lambda s}\left[\lambda \ln V(X(s))+a_{1}(r(s))+b_{1}(r(s))\right. \\
+ & a_{2}(r(s))+b_{2}(r(s))+a_{3}(r(s))+\frac{e_{3}(r(s))}{f_{1}(r(s))} \\
+ & \frac{e_{4}(r(s))}{f_{2}(r(s))}-\frac{(1-\epsilon) m_{1} \sigma_{2}^{2}(r(s))}{2} x^{2} \\
- & \frac{(1-\epsilon) m_{2} \sigma_{4}^{2}(r(s))}{2} y^{2} \\
- & \left.\frac{(1-\epsilon) m_{3} \sigma_{6}^{2}(r(s))}{2} z^{2}\right] \mathrm{d} s,
\end{aligned}
$$

for all $0 \leq t \leq k$ and $k \geq k_{0}(\omega)$. It is obvious that there is a positive constant $\mathrm{H}_{3}$ such that

$$
\begin{aligned}
\lambda \ln V & (X(t))+a_{1}(r(t))+b_{1}(r(t))+a_{2}(r(t)) \\
& +b_{2}(r(t))+a_{3}(r(t))+\frac{e_{3}(r(t))}{f_{1}(r(t))}+\frac{e_{4}(r(t))}{f_{2}(r(t))} \\
& -\frac{(1-\epsilon) m_{1} \sigma_{2}^{2}(r(t))}{2} x^{2} \\
- & \frac{(1-\epsilon) m_{2} \sigma_{4}^{2}(r(t))}{2} y^{2} \\
- & \frac{(1-\epsilon) m_{3} \sigma_{6}^{2}(r(t))}{2} z^{2} \leq H_{3} .
\end{aligned}
$$

Then

$$
e^{\lambda t} \ln V(X(t)) \leq \ln V(X(0))+\frac{\eta e^{\lambda k}}{\epsilon} \ln k+\frac{H_{3}}{\lambda} e^{\lambda t}
$$

for all $0 \leq t \leq k$ and $k \geq k_{0}(\omega)$. Thus, for any $\omega \in \Omega_{0}$, if $k-1 \leq t \leq k(k>2)$ and $k \geq k_{0}(\omega)$, we have

$$
\begin{aligned}
& \frac{\ln V(X(t))}{\ln t} \leq \frac{1}{\ln (k-1)}\left[e^{-\lambda(k-1)} \ln V(X(0))\right. \\
& \left.+\frac{\eta e^{\lambda}}{\epsilon} \ln k+\frac{H_{3}}{\lambda}\right] .
\end{aligned}
$$

This implies

$$
\limsup _{t \rightarrow \infty} \frac{\ln V(X(t))}{\ln t} \leq \frac{\eta e^{\lambda}}{\epsilon} \text { a.s. }
$$

Let $\epsilon \rightarrow 1, \eta \rightarrow 1$, and $\lambda \rightarrow 0$, so we get $\lim \sup _{t \rightarrow \infty}(\ln V(X(t)) / \ln t) \leq 1$ a.s. Then we can directly get the desired conclusion.

\section{Examples and Numerical Simulations}

In this section, we will give two examples and make some numerical simulations to support main results. By the method mentioned in [26], the discrete form of system (5) can be given by

$$
\begin{aligned}
& x_{n+1}=x_{n}+x_{n}\left(a_{1}\left(r_{n}\right)+\frac{b_{1}\left(r_{n}\right) y_{n-m_{1}}}{k_{1}\left(r_{n}\right)+y_{n-m_{1}}}\right. \\
& \left.-c_{1}\left(r_{n}\right) x_{n}-\frac{e_{1}\left(r_{n}\right) z_{n}}{1+f_{1}\left(r_{n}\right) x_{n}}\right) \Delta t+\sigma_{1}\left(r_{n}\right) \\
& \cdot x_{n} \sqrt{\Delta t} \zeta_{1 n}+\sigma_{2}\left(r_{n}\right) x_{n}^{2} \sqrt{\Delta t} \zeta_{2 n}+\frac{\sigma_{1}^{2}\left(r_{n}\right)}{2} x_{n}\left(\zeta_{1 n}^{2}\right. \\
& -1) \Delta t+\sigma_{2}^{2}\left(r_{n}\right) x_{n}^{3}\left(\zeta_{2 n}^{2}-1\right) \Delta t,
\end{aligned}
$$



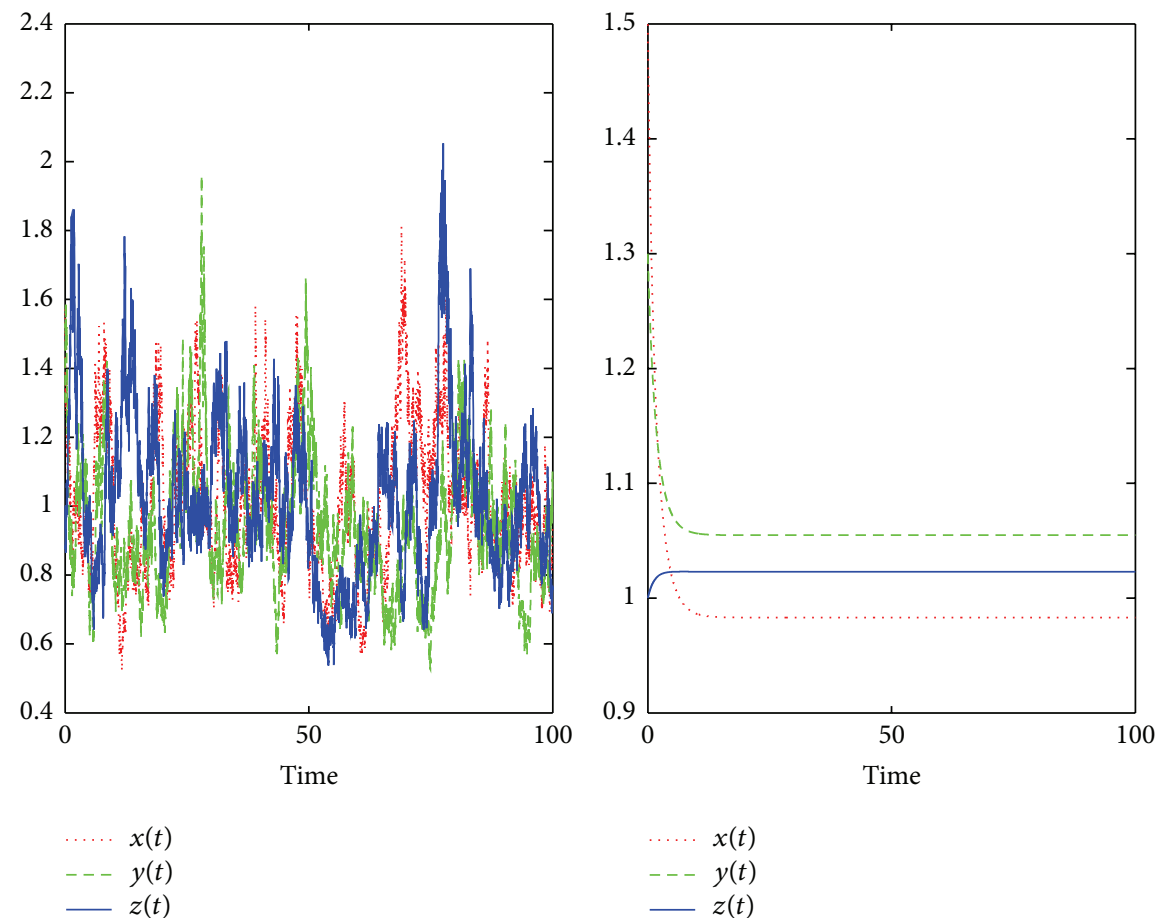

FIGURE 1: (Stochastic permanence) the trajectories of the solution $(x(t), y(t), z(t))$ for subsystem $(64)$ and its corresponding deterministic system.

$$
\begin{aligned}
& y_{n+1}=y_{n}+y_{n}\left(a_{2}\left(r_{n}\right)+\frac{b_{2}\left(r_{n}\right) x_{n-m_{2}}}{k_{2}\left(r_{n}\right)+x_{n-m_{2}}}-c_{2}\left(r_{n}\right) y_{n}\right. \\
& \left.-\frac{e_{2}\left(r_{n}\right) z_{n}}{1+f_{2}\left(r_{n}\right) y_{n}+f_{3}\left(r_{n}\right) z_{n}}\right) \Delta t+\sigma_{3}\left(r_{n}\right) \\
& \cdot y_{n} \sqrt{\Delta t} \zeta_{3 n}+\sigma_{4}\left(r_{n}\right) y_{n}^{2} \sqrt{\Delta t} \zeta_{4 n}+\frac{\sigma_{3}^{2}\left(r_{n}\right)}{2} y_{n}\left(\zeta_{3 n}^{2}\right. \\
& -1) \Delta t+\sigma_{4}^{2}\left(r_{n}\right) y_{n}^{3}\left(\zeta_{4 n}^{2}-1\right) \Delta t, \\
& z_{n+1}=z_{n}+z_{n}\left(a_{3}\left(r_{n}\right)+\frac{e_{3}\left(r_{n}\right) x_{n-m_{3}}}{1+f_{1}\left(r_{n}\right) x_{n-m_{3}}}\right. \\
& \left.+\frac{e_{4}\left(r_{n}\right) y_{n-m_{4}}}{1+f_{2}\left(r_{n}\right) y_{n-m_{4}}+f_{3}\left(r_{n}\right) z_{n-m_{4}}}-c_{3}\left(r_{n}\right) z_{n}\right) \\
& +\frac{\sigma_{5}^{2}\left(r_{n}\right)}{2} z_{n}\left(\zeta_{5 n}^{2}-1\right) \Delta t+\sigma_{6}^{2}\left(r_{n}\right) z_{n}^{3}\left(\zeta_{6 n}^{2}-1\right) \Delta t \\
& +\sigma_{n} \sqrt{\Delta t} \zeta_{5 n}+\sigma_{6}\left(r_{n}\right) z_{n}^{2} \sqrt{\Delta t} \zeta_{6 n}
\end{aligned}
$$

where $\zeta_{j n}, j=1,2, \ldots, 6$, are Gaussian random variables that follow $N(0,1)$ and $m_{j}$ denotes the integer part of $\tau_{j} / \Delta t-$ $1, j=1,2,3,4$. For the procedure of generating the discrete Markov chain $\left\{r_{n}, n=0,1,2, \ldots\right\}$, please refer to [18].

Throughout this section, we assume that $\Delta t=0.01, \tau_{1}=$ $0.1, \tau_{2}=0.2, \tau_{3}=0.3, \tau_{4}=0.4$, and let the initial data be $x(\theta)=1.5, y(\theta)=1.3, z(\theta)=1, r(0)=1, \theta \in[-\tau, 0]$, where $\tau=\max \left\{\tau_{1}, \tau_{2}, \tau_{3}, \tau_{4}\right\}$. Please note that in this section all of numerical results are computed by MATLAB and displayed in rational format. Therefore there may be an error for some numerical results.

Example 1. Let $r(t)$ be a right-continuous Markov chain taking values in $\mathbb{S}=\{1,2\}$. System (5) may be regarded as the result of the following two subsystems:

$\mathrm{d} x(t)$

$$
\begin{aligned}
& =x\left(0.5+\frac{0.1 y_{\tau_{1}}}{3+y_{\tau_{1}}}-0.5 x-\frac{0.1 z}{1+2 x}\right) \mathrm{d} t \\
& +0.2 x \mathrm{~d} B_{1}(t)+0.1 x^{2} \mathrm{~d} B_{2}(t),
\end{aligned}
$$

$\mathrm{d} y(t)$

$$
\begin{aligned}
= & y\left(0.6+\frac{0.25 x_{\tau_{2}}}{2+x_{\tau_{2}}}-0.6 y-\frac{0.2 z}{1+2 y+z}\right) \mathrm{d} t \\
& +0.2 y \mathrm{~d} B_{3}(t)+0.1 y^{2} \mathrm{~d} B_{4}(t),
\end{aligned}
$$

$\mathrm{d} z(t)$

$$
\begin{aligned}
& =z\left(0.7+\frac{0.01 x_{\tau_{3}}}{1+2 x_{\tau_{3}}}+\frac{0.05 y_{\tau_{4}}}{1+2 y_{\tau_{4}}+z_{\tau_{4}}}-0.7 z\right) \mathrm{d} t \\
& \quad+0.2 z \mathrm{~d} B_{5}(t)+0.1 z^{2} \mathrm{~d} B_{6}(t),
\end{aligned}
$$



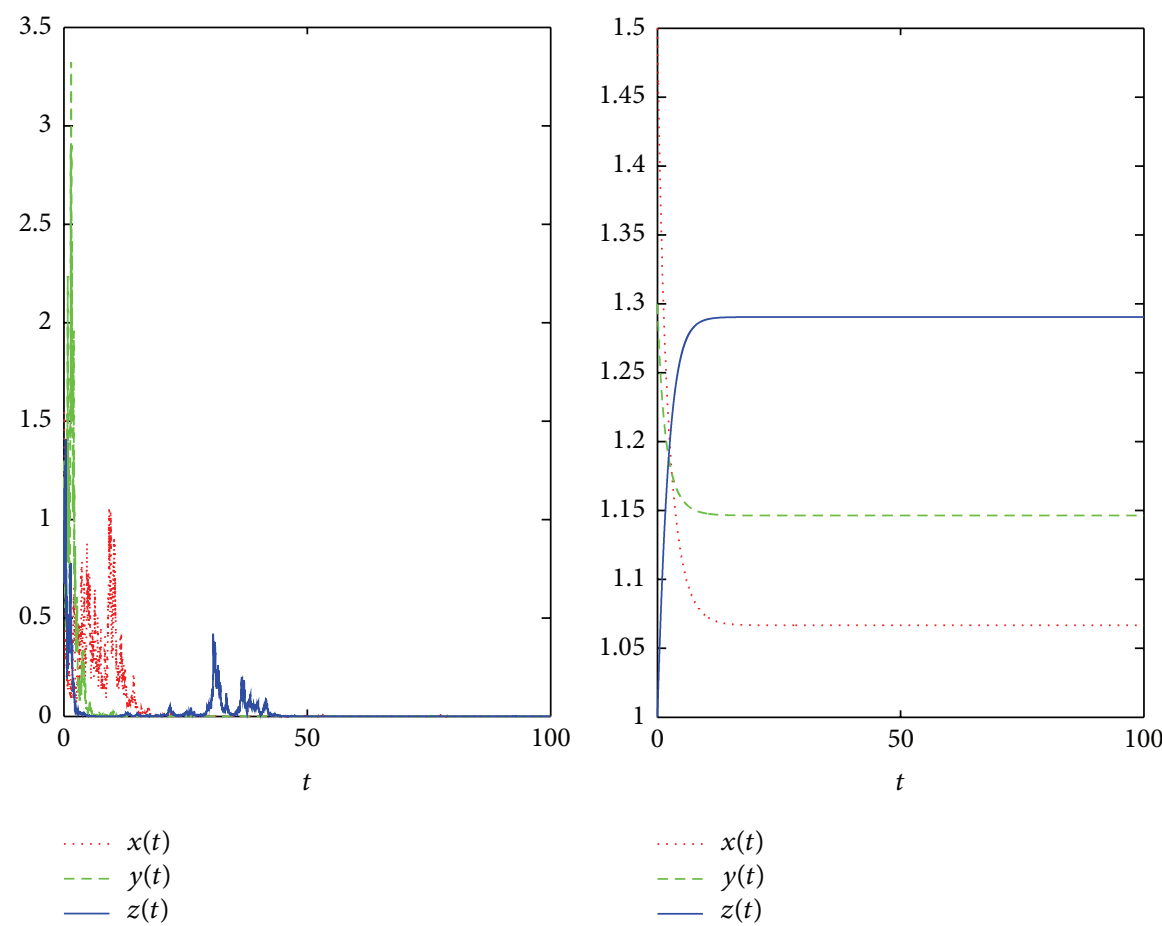

FIGURE 2: (Extinction) the trajectories of the solution $(x(t), y(t), z(t))$ for subsystem (65) and its corresponding deterministic system.

$\mathrm{d} x(t)$

$$
\begin{aligned}
= & x\left(0.45+\frac{0.2 y_{\tau_{1}}}{1+y_{\tau_{1}}}-0.45 x-\frac{0.25 z}{1+3 x}\right) \mathrm{d} t \\
& +1.2 x \mathrm{~d} B_{1}(t)+0.05 x^{2} \mathrm{~d} B_{2}(t),
\end{aligned}
$$

$\mathrm{d} y(t)$

$$
\begin{aligned}
= & y\left(0.75+\frac{0.3 x_{\tau_{2}}}{1+x_{\tau_{2}}}-0.75 y-\frac{0.2 z}{1+3 y+z}\right) \mathrm{d} t \\
& +1.5 y \mathrm{~d} B_{3}(t)+0.05 y^{2} \mathrm{~d} B_{4}(t),
\end{aligned}
$$

$\mathrm{d} z(t)$

$$
\begin{aligned}
= & z\left(0.5+\frac{0.04 x_{\tau_{3}}}{1+3 x_{\tau_{3}}}+\frac{0.03 y_{\tau_{4}}}{1+3 y_{\tau_{4}}+z_{\tau_{4}}}-0.4 z\right) \mathrm{d} t \\
& +1.1 z \mathrm{~d} B_{5}(t)+0.05 z^{2} \mathrm{~d} B_{6}(t),
\end{aligned}
$$

switching from one to another according to the movement of Markov chain $r(t)$.

Then we compute that $q(1)=12 / 25>0, a_{1}(1)+b_{1}(1)-$ $(1 / 2) \sigma_{1}^{2}(1)=29 / 50, a_{2}(1)+b_{2}(1)-(1 / 2) \sigma_{3}^{2}(1)=83 / 100$, $a_{3}(1)+e_{3}(1) / f_{1}(1)+e_{4}(1) / f_{2}(1)-(1 / 2) \sigma_{5}^{2}(1)=71 / 100$. Therefore, by Corollary 9 , subsystem (64) is stochastically permanent. See Figure 1.

Compute also $q(2)=-31 / 40, a_{1}(2)+b_{1}(2)-(1 / 2) \sigma_{1}^{2}(2)=$ $-7 / 100<0, a_{2}(2)+b_{2}(2)-(1 / 2) \sigma_{3}^{2}(2)=-3 / 40<0$, $a_{3}(2)+e_{3}(2) / f_{1}(2)+e_{4}(2) / f_{2}(2)-(1 / 2) \sigma_{5}^{2}(2)=-49 / 600<0$. Therefore, by Corollary 11, subsystem (65) is extinct. See Figure 2.

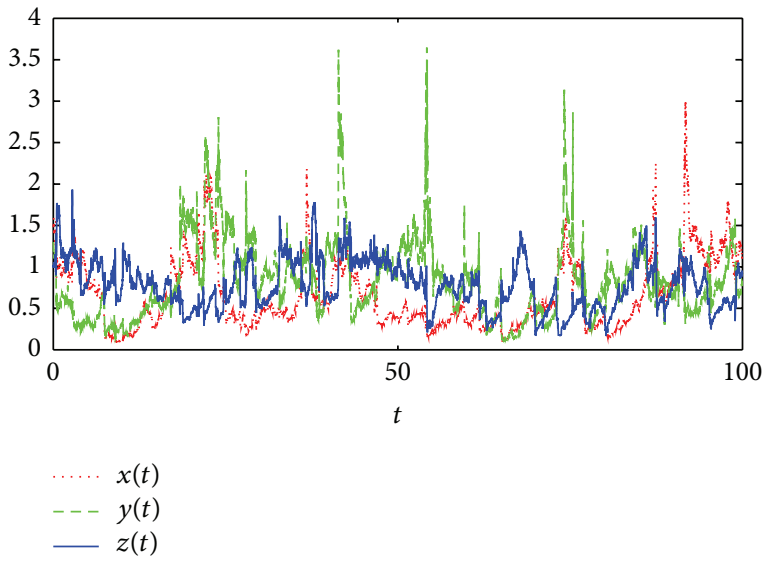

FIGURE 3: (Stochastic permanence) the trajectories of the solution $(x(t), y(t), z(t))$ for the overall system (5) in Case 1 of Example 1.

Case 1. Assume that the generator of Markov chain $r(t)$ is

$$
\Gamma=\left[\begin{array}{cc}
-1 & 1 \\
8 & -8
\end{array}\right] \text {. }
$$

By solving (11), we get the unique stationary distribution

$$
\pi=\left(\frac{8}{9}, \frac{1}{9}\right) \text {. }
$$

Then compute that $\sum_{i=1}^{N} \pi_{i} q(i)=\sum_{i=1}^{N} \pi_{i}\left(\min \left\{a_{1}(i), a_{2}(i), a_{3}(i)-\right.\right.$ $\left.\left.e_{1}(i) / f_{1}(i)-e_{2}(i) / f_{2}(i)\right\}-(1 / 2) \max \left\{\sigma_{1}^{2}(i), \sigma_{3}^{2}(i), \sigma_{5}^{2}(i)\right\}\right)=$ $(12 / 25) \pi_{1}-(31 / 40) \pi_{2}=613 / 1800>0$. Therefore, by Theorem 8 , the overall system (5) is stochastically permanent. See Figures 3-5. 


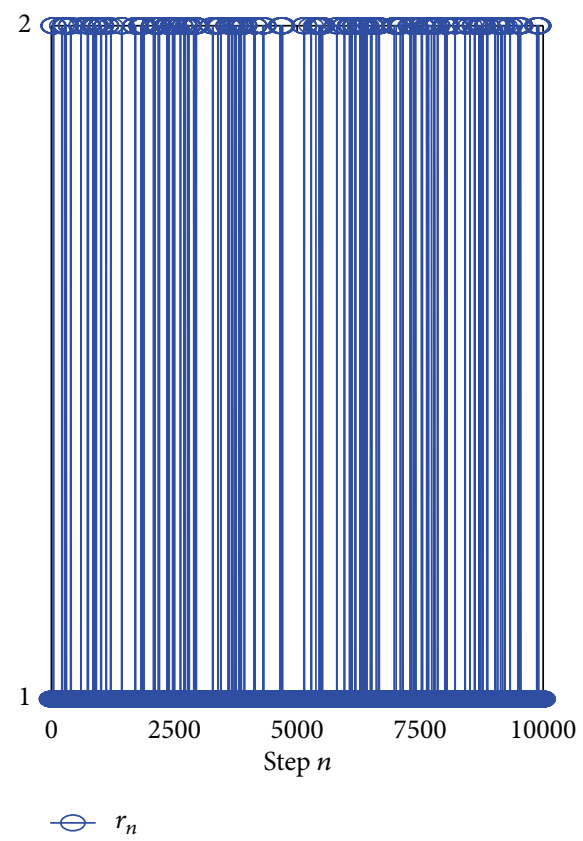

(a)

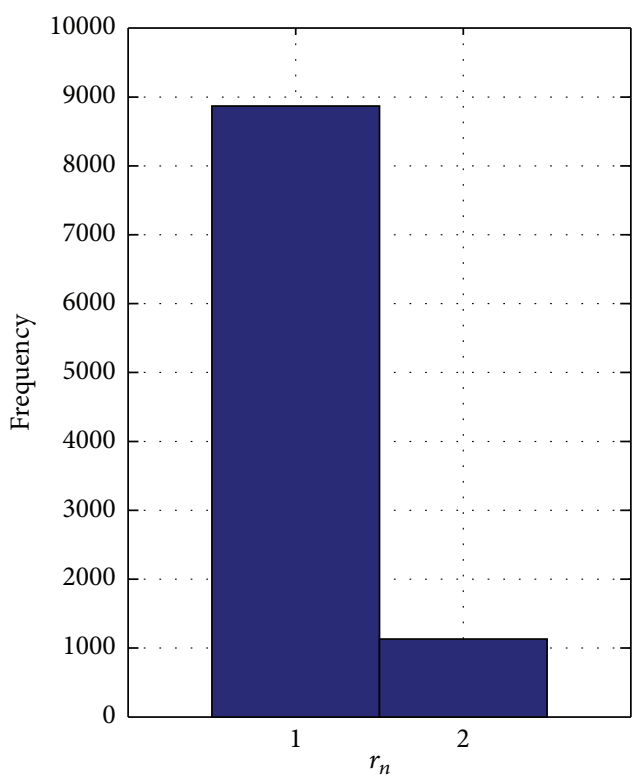

(b)

FIGURE 4: The subgraphs (a) and (b) denote the trajectory and frequency of the discrete Markov chain $r_{n}$ taking value in $\{1,2\}$, respectively. $r_{n}=1$ and $r_{n}=2$ mean that the overall system (5) switches to subsystems (64) and (65) in step $n$, respectively. The change of $r_{n}$ in (a) means the process of regime switching of system (5) between subsystems (64) and (65). And the frequency of $r_{n}$ in (b) shows the result of regime switching - the total number of steps of the overall system (5) switching to subsystem (64) or subsystem (65). This graph shows that under the control of the Markov chain the overall system (5) mostly switches to subsystem (64) in Case 1 of Example 1.

Case 2. Assume that the generator of Markov chain $r(t)$ is

$$
\Gamma=\left[\begin{array}{cc}
-12 & 12 \\
1 & -1
\end{array}\right]
$$

By solving (11), we get the unique stationary distribution

$$
\pi=\left(\frac{1}{13}, \frac{12}{13}\right)
$$

Then we compute that $\sum_{i \in \mathbb{S}} \pi_{i}\left(a_{1}(i)+b_{1}(i)-(1 / 2) \sigma_{1}^{2}(i)\right)=$ $(29 / 50) \pi_{1}-(7 / 100) \pi_{2}=-1 / 50<0, \sum_{i \in \mathbb{S}} \pi_{i}\left(a_{2}(i)+\right.$ $\left.b_{2}(i)-(1 / 2) \sigma_{3}^{2}(i)\right)=(83 / 100) \pi_{1}-(3 / 40) \pi_{2}=-7 / 1300<$ $0, \sum_{i \in \mathbb{S}} \pi_{i}\left(a_{3}(i)+e_{3}(i) / f_{1}(i)+e_{4}(i) / f_{2}(i)-(1 / 2) \sigma_{5}^{2}(i)\right)=$ $(71 / 100) \pi_{1}-(49 / 600) \pi_{2}=-27 / 1300<0$. Therefore, by Theorem 10, the overall system (5) is extinct. See Figures 6-8.

Example 2. Let $r(t)$ be a right-continuous Markov chain taking values in $\mathbb{S}=\{1,2,3\}$. System (5) may be regarded as the result of the following three subsystems:

$$
\begin{aligned}
& \mathrm{d} x(t) \\
& =x\left(0.5+\frac{0.1 y_{\tau_{1}}}{3+y_{\tau_{1}}}-0.5 x-\frac{0.1 z}{1+2 x}\right) \mathrm{d} t \\
& \quad+1.2 x \mathrm{~d} B_{1}(t)+0.1 x^{2} \mathrm{~d} B_{2}(t),
\end{aligned}
$$

$$
\begin{aligned}
& \mathrm{d} y(t) \\
& =y\left(0.6+\frac{0.25 x_{\tau_{2}}}{2+x_{\tau_{2}}}-0.6 y-\frac{0.2 z}{1+2 y+z}\right) \mathrm{d} t \\
& \quad+1.4 y \mathrm{~d} B_{3}(t)+0.1 y^{2} \mathrm{~d} B_{4}(t), \\
& \mathrm{d} z(t) \\
& =z\left(0.7+\frac{0.01 x_{\tau_{3}}}{1+2 x_{\tau_{3}}}+\frac{0.05 y_{\tau_{4}}}{1+2 y_{\tau_{4}}+z_{\tau_{4}}}-0.7 z\right) \mathrm{d} t \\
& \quad+1.3 z \mathrm{~d} B_{5}(t)+0.1 z^{2} \mathrm{~d} B_{6}(t),
\end{aligned}
$$

$\mathrm{d} x(t)$

$$
\begin{aligned}
= & x\left(0.3+\frac{0.05 y_{\tau_{1}}}{3+y_{\tau_{1}}}-0.5 x-\frac{0.1 z}{1+8 x}\right) \mathrm{d} t \\
& +1.1 x \mathrm{~d} B_{1}(t)+0.06 x^{2} \mathrm{~d} B_{2}(t),
\end{aligned}
$$

$\mathrm{d} y(t)$

$$
\begin{aligned}
= & y\left(0.2+\frac{0.1 x_{\tau_{2}}}{2+x_{\tau_{2}}}-0.4 y-\frac{0.1 z}{1+7 y+z}\right) \mathrm{d} t \\
& +1.05 y \mathrm{~d} B_{3}(t)+0.06 y^{2} \mathrm{~d} B_{4}(t),
\end{aligned}
$$

$\mathrm{d} z(t)$

$$
\begin{aligned}
= & z\left(0.1+\frac{0.08 x_{\tau_{3}}}{1+8 x_{\tau_{3}}}+\frac{0.07 y_{\tau_{4}}}{1+7 y_{\tau_{4}}+z_{\tau_{4}}}-0.3 z\right) \mathrm{d} t \\
& +0.9 z \mathrm{~d} B_{5}(t)+0.08 z^{2} \mathrm{~d} B_{6}(t),
\end{aligned}
$$




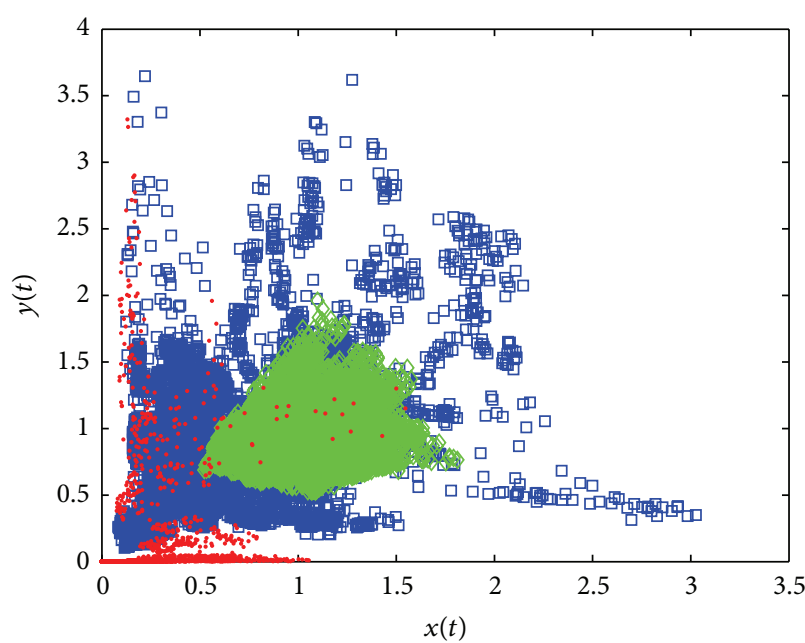

ㅁ (5)

$\diamond(64)$

- (65)

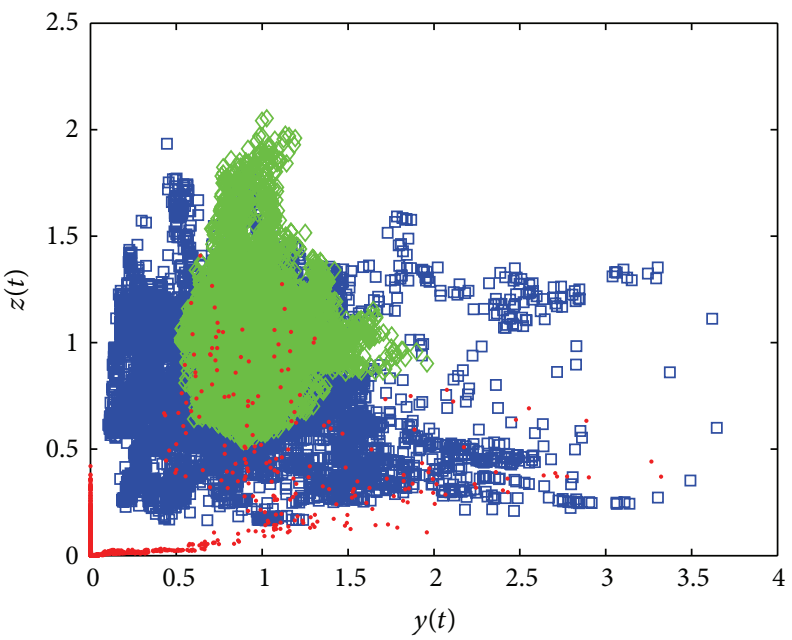

ㅁ (5)

$\diamond(64)$

- (65)

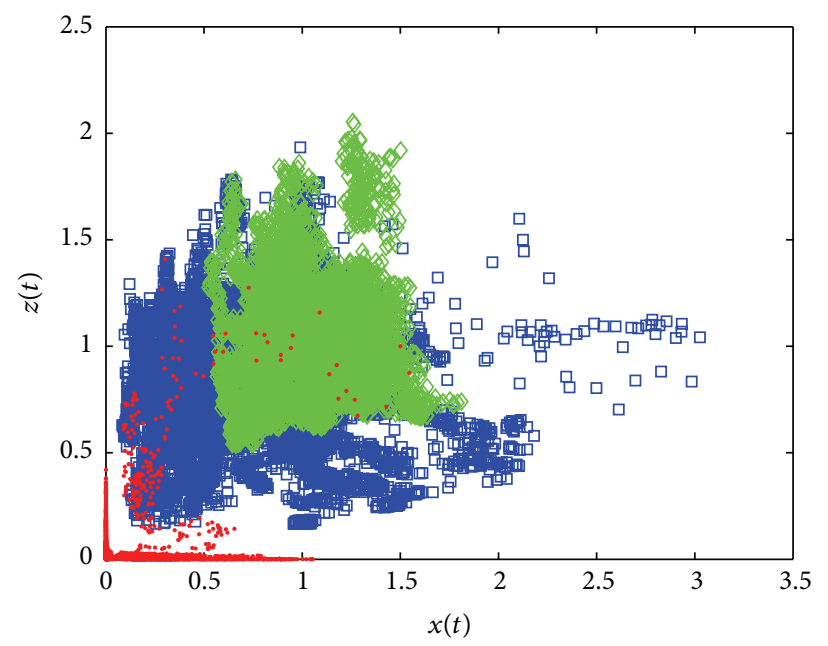

ㅁ (5)

$\diamond(64)$

- (65)

(b)

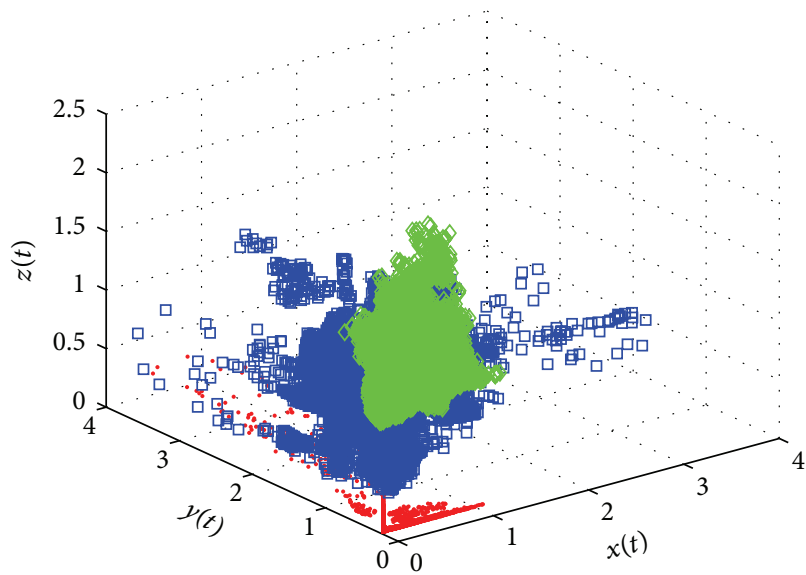

ㅁ (5)

$\diamond(64)$

- (65)

(c)

(d)

Figure 5: The subgraphs (a), (b), (c), and (d) denote the discrete point distribution of two subsystems and the overall system in $x y, x z, y z$, and $x y z$, respectively. The blue, green, and red areas represent the overall system (5), subsystem (64), and subsystem (65), respectively. The green area which means stochastic permanence is far away from the origin while most points of the red area which means extinction lie in the origin. Under the control of Markov chain, the blue area also keeps away from the origin and means stochastic permanence in Case 1 of Example 1.

$\mathrm{d} x(t)$

$$
\begin{aligned}
= & x\left(0.75+\frac{0.01 y_{\tau_{1}}}{3+y_{\tau_{1}}}-0.75 x-\frac{0.3 z}{1+6 x}\right) \mathrm{d} t \\
& +0.4 x \mathrm{~d} B_{1}(t)+0.1 x^{2} \mathrm{~d} B_{2}(t),
\end{aligned}
$$

$\mathrm{d} y(t)$

$$
=y\left(0.7+\frac{0.01 x_{\tau_{2}}}{3+x_{\tau_{2}}}-0.68 y-\frac{0.4 z}{1+8 y+3 z}\right) \mathrm{d} t
$$

$$
+0.4 y \mathrm{~d} B_{3}(t)+0.1 y^{2} \mathrm{~d} B_{4}(t)
$$

$\mathrm{d} z(t)$

$$
=z\left(0.68+\frac{0.04 x_{\tau_{3}}}{1+6 x_{\tau_{3}}}+\frac{0.06 y_{\tau_{4}}}{1+8 y_{\tau_{4}}+3 z_{\tau_{4}}}-0.7 z\right) \mathrm{d} t
$$$$
+0.4 z \mathrm{~d} B_{5}(t)+0.1 z^{2} \mathrm{~d} B_{6}(t),
$$ 

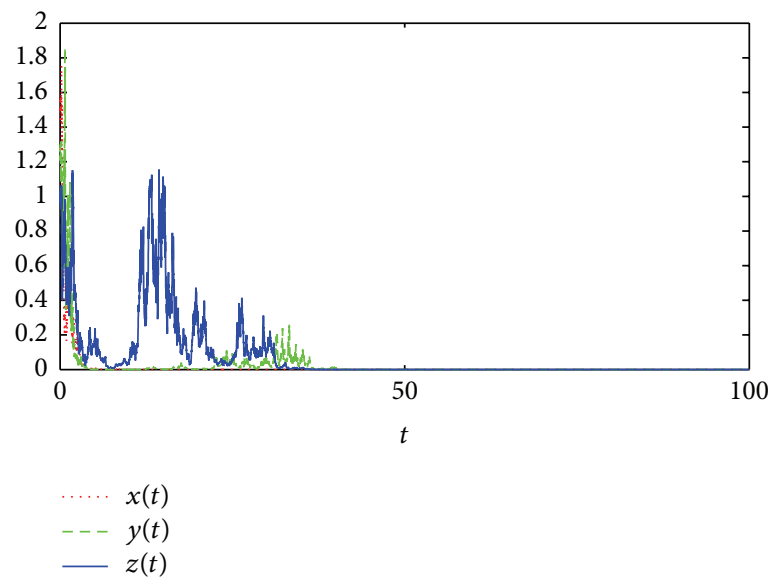

Figure 6: (Extinction) the trajectories of the solution $(x(t), y(t), z(t))$ for the overall system (5) in Case 2 of Example 1.

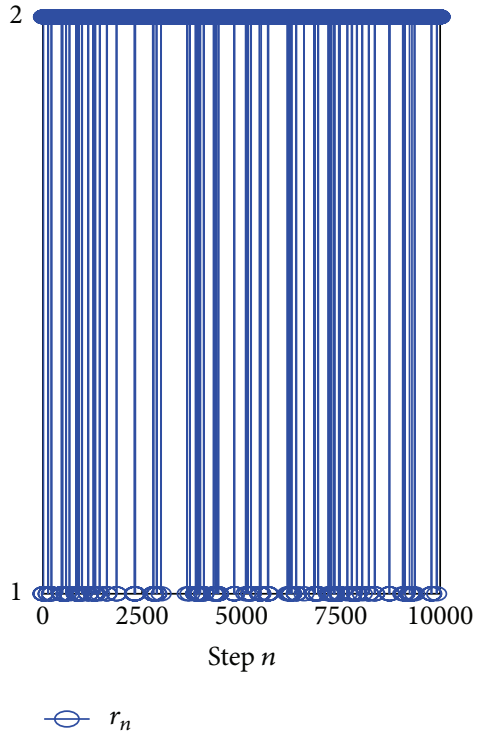

(a)

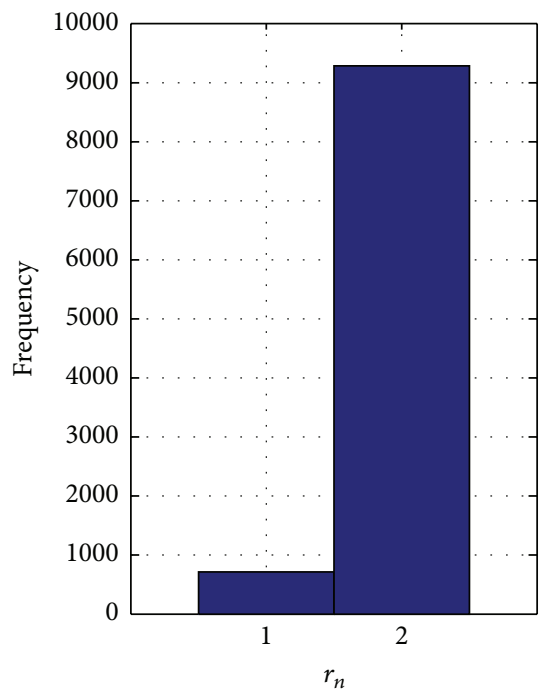

(b)

Figure 7: The above subgraphs have the same notations as in Figure 4. The change of $r_{n}$ in (a) means the process of regime switching of system (5) between subsystems (64) and (65). And the frequency of $r_{n}$ in (b) shows the result of regime switching-the total number of steps of the overall system (5) switching to subsystem (64) or subsystem (65). This graph means that under the control of the Markov chain the overall system (5) mostly switches to subsystem (65) in Case 2 of Example 1.

switching from one to another according to the movement of Markov chain $r(t)$.

We compute that $q(1)=-12 / 25, a_{1}(1)+b_{1}(1)-$ $(1 / 2) \sigma_{1}^{2}(1)=-3 / 25<0, a_{2}(1)+b_{2}(1)-(1 / 2) \sigma_{3}^{2}(1)=$ $-13 / 100<0, a_{3}(1)+e_{3}(1) / f_{1}(1)+e_{4}(1) / f_{2}(1)-(1 / 2) \sigma_{5}^{2}(1)=$ $-23 / 200<0$. Therefore, by Corollary 11 , subsystem (70) is extinct.

Compute $q(2)=-527 / 991, a_{1}(2)+b_{1}(2)-(1 / 2) \sigma_{1}^{2}(2)=$ $-51 / 200<0, a_{2}(2)+b_{2}(2)-(1 / 2) \sigma_{3}^{2}(2)=-201 / 800<0$, $a_{3}(2)+e_{3}(2) / f_{1}(2)+e_{4}(2) / f_{2}(2)-(1 / 2) \sigma_{5}^{2}(2)=-57 / 200<0$. Therefore, by Corollary 11, subsystem (71) is extinct.

Compute again $q(3)=1 / 2>0, a_{1}(3)+b_{1}(3)-$ $(1 / 2) \sigma_{1}^{2}(3)=17 / 25, a_{2}(3)+b_{2}(3)-(1 / 2) \sigma_{3}^{2}(3)=63 / 100$, $a_{3}(3)+e_{3}(3) / f_{1}(3)+e_{4}(3) / f_{2}(3)-(1 / 2) \sigma_{5}^{2}(3)=737 / 1200$.
Therefore, by Corollary 9, subsystem (72) is stochastically permanent.

Case 1. Assume that the generator of the Markov chain $r(t)$ is

$$
\Gamma=\left[\begin{array}{ccc}
-11 & 1 & 10 \\
3 & -3 & 0 \\
1 & 0 & -1
\end{array}\right]
$$

By solving (11), we get the unique stationary distribution

$$
\pi=\left(\frac{3}{34}, \frac{1}{34}, \frac{15}{17}\right)
$$



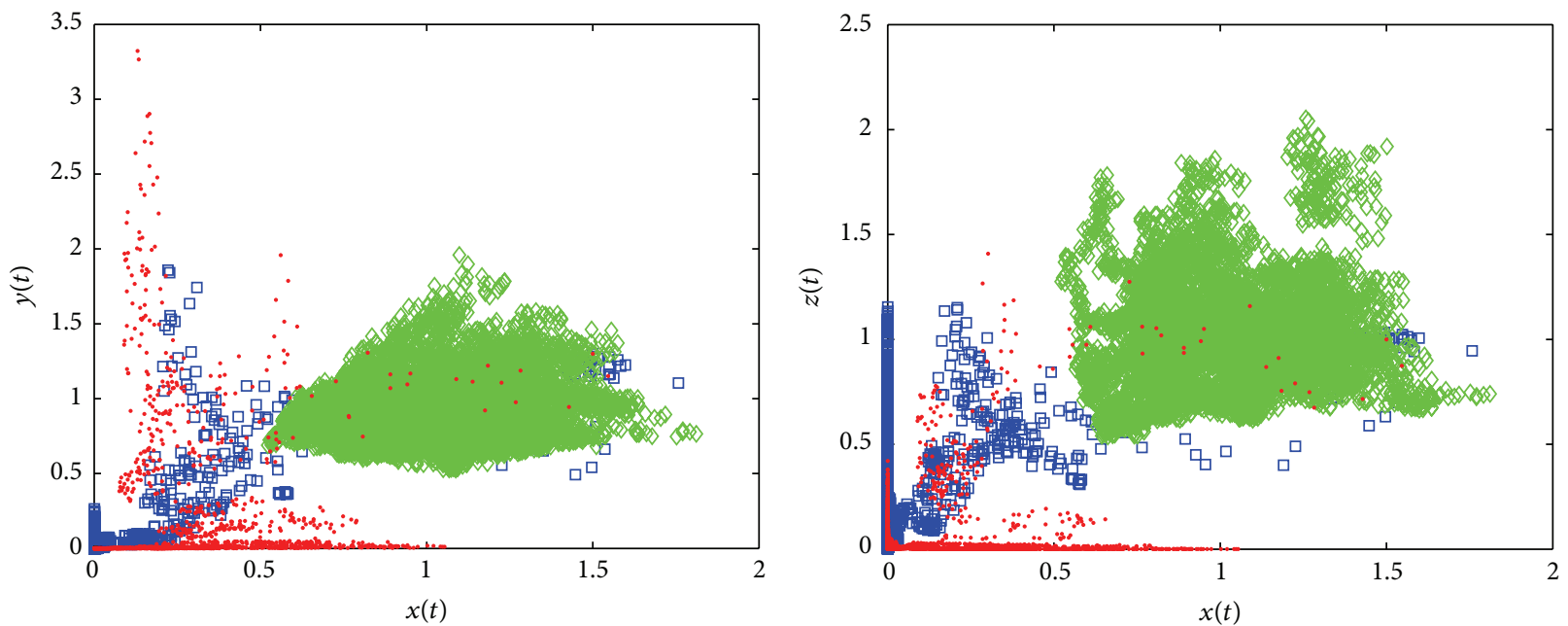
ㅁ (5)
ㅁ (5)
$\diamond(64)$
- (65)
$\diamond(64)$
- (65)

(a)

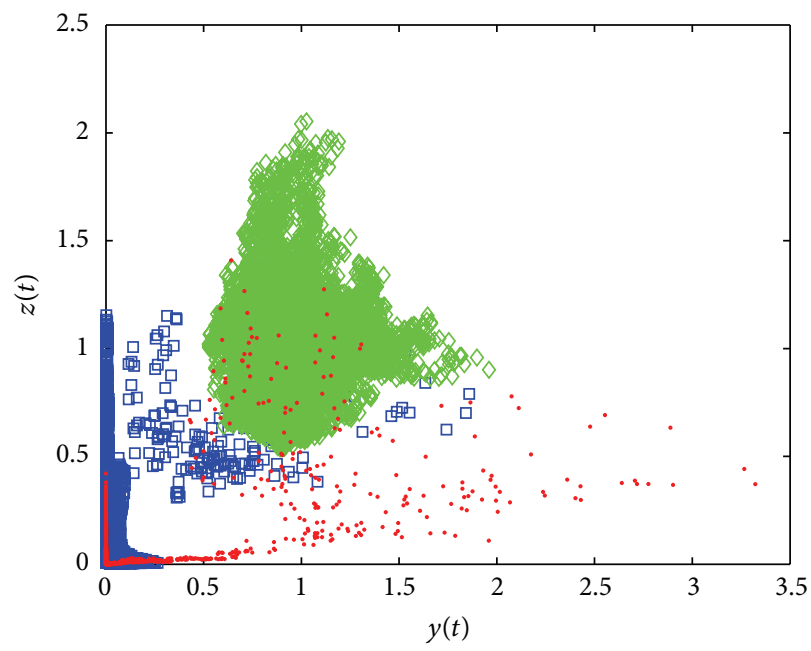

(b)

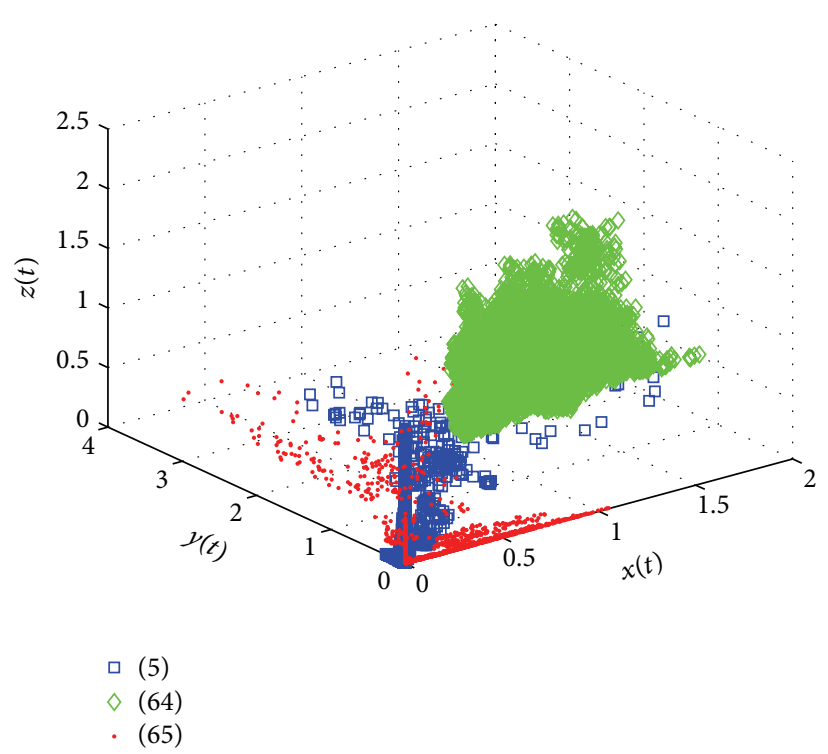

$\diamond(64)$

$(65)$

(c)

(d)

Figure 8: The above subgraphs have the same notations as in Figure 5. Under the control of Markov chain, most points of the blue area lie in the origin and mean extinction in Case 2 of Example 1.

Then compute that $\sum_{i=1}^{N} \pi_{i} q(i)=\sum_{i=1}^{N} \pi_{i}\left(\min \left\{a_{1}(i), a_{2}(i)\right.\right.$, $\left.\left.a_{3}(i)-e_{1}(i) / f_{1}(i)-e_{2}(i) / f_{2}(i)\right\}-(1 / 2) \max \left\{\sigma_{1}^{2}(i), \sigma_{3}^{2}(i), \sigma_{5}^{2}(i)\right\}\right)=$ $-(12 / 25) \pi_{1}-(527 / 991) \pi_{2}+(1 / 2) \pi_{3}=679 / 1772>0$. Therefore, by Theorem 8 , the overall system (5) is stochastically permanent.

Case 2. Assume that the generator of the Markov chain $r(t)$ is

$$
\Gamma=\left[\begin{array}{ccc}
-3 & 2 & 1 \\
1 & -1 & 0 \\
2 & 0 & -2
\end{array}\right] \text {. }
$$

By solving (11), we get the unique stationary distribution

$$
\pi=\left(\frac{2}{7}, \frac{4}{7}, \frac{1}{7}\right) .
$$

Then we compute that $\sum_{i \in \mathbb{S}} \pi_{i}\left(a_{1}(i)+b_{1}(i)-(1 / 2) \sigma_{1}^{2}(i)\right)=$ $-(3 / 25) \pi_{1}-(51 / 200) \pi_{2}+(17 / 25) \pi_{3}=-29 / 350<$ $0, \sum_{i \in \mathbb{S}} \pi_{i}\left(a_{2}(i)+b_{2}(i)-(1 / 2) \sigma_{3}^{2}(i)\right)=-(13 / 100) \pi_{1}-$ $(201 / 800) \pi_{2}+(63 / 100) \pi_{3}=-127 / 1400<0, \sum_{i \in \mathbb{S}} \pi_{i}\left(a_{3}(i)+\right.$ $\left.e_{3}(i) / f_{1}(i)+e_{4}(i) / f_{2}(i)-(1 / 2) \sigma_{5}^{2}(i)\right)=-(23 / 200) \pi_{1}-$ $(57 / 200) \pi_{2}+(737 / 1200) \pi_{3}=-199 / 1843<0$. Therefore, by Theorem 10, the overall system (5) is extinct. 
Case 3. Now we only consider the effect of white noise's intensities. From Examples 1 and 2, we know that subsystem (64) is stochastically permanent while subsystem (70) is extinct. Note that the only difference between subsystems (64) and (70) is the intensity of white noise, that is, $\sigma_{1}, \sigma_{3}$, $\sigma_{5}$. In other words, large white noise leads to extinction.

\section{Discussion and Conclusion}

In this paper, we investigate dynamical behavior of a stochastic delayed one-predator and two-mutualistic-prey model perturbed by white noise and telegraph noise.

Theorems 8 and 10 show us sufficient conditions of stochastic permanence and extinction for system (5), respectively. These conditions are all dependent on both parameters of each subsystem (7) and the stationary distribution probability. This means that under the control of Markov chain, if some subsystems are stochastically permanent while others are extinct, the overall system (5) is stochastically permanent or extinct, determined by the sign of $\sum_{i=1}^{N} \pi_{i} q(i)$ and $\sum_{i \in \mathbb{S}} \pi_{i}\left(a_{1}(i)+b_{1}(i)-(1 / 2) \sigma_{1}^{2}(i)\right), \quad \sum_{i \in \mathbb{S}} \pi_{i}\left(a_{2}(i)+\right.$ $\left.b_{2}(i)-(1 / 2) \sigma_{3}^{2}(i)\right), \sum_{i \in \mathbb{S}} \pi_{i}\left(a_{3}(i)+e_{3}(i) / f_{1}(i)+e_{4}(i) / f_{2}(i)-\right.$ $\left.(1 / 2) \sigma_{5}^{2}(i)\right)$. This explanation can be verified by Cases $1-2$ of Example 1 or Cases 1-2 of Example 2.

Corollaries 9 and 11 also give sufficient conditions of stochastic permanence and extinction for subsystem (7), respectively. These two corollaries tell us that large white noise can cause extinction while relatively small one can make system stochastically permanent; see Case 3 of Example 2.

It is worth noting that the traditional definition of stochastic permanence (Definition 2) has weakness: even some species are extinctive; the model could be stochastic permanent. Some authors have introduced a more appropriate definition of permanence for stochastic population model, that is, stochastically persistent in probability (e.g., see $[27,28]$ ), which is a more appropriate definition of permanence than stochastic permanence. We are interested in investigating stochastic persistence in probability in the future.

Beside the one-predator and two-mutualistic-prey model, there are other three-species models, for example, tri-trophic food-chain model and two-prey one-predator model; please see [29-31]. At the same time, beside white noise and telegraph noise, Lévy noise is inevitable in the nature. And there are some researches about stochastic population models with Lévy noise; please see [31-33]. Therefore, the above threespecies models with Lévy noise deserve further investigation and we may consider them in the future.

\section{Competing Interests}

The authors declare that there is no conflict of interests regarding the publication of this paper.

\section{Acknowledgments}

The authors would like to thank, for financial support, the National Natural Science Foundation of China (nos. 61563033 and 11563005), the Natural Science Foundation of Jiangxi Province (nos. 20122BAB201002 and 20151BAB212011), and the Innovation Fund Designated for Graduate Students of Nanchang University (no. cx2015089).

\section{References}

[1] R. May, Theoretical Ecology: Principles and Applications, WB Saunders Company, Philadelphia, Pa, USA, 1976.

[2] A. M. Dean, "A simple model of mutualism," The American Naturalist, vol. 121, no. 3, pp. 409-417, 1983.

[3] C. L. Wolin and L. R. Lawlor, "Models of facultative mutualism: density effects," The American Naturalist, vol. 124, no. 6, pp. 843$862,1984$.

[4] D. H. Boucher, "Lotka-volterra models of mutualism and positive density-dependence," Ecological Modelling, vol. 27, no. 3-4, pp. 251-270, 1985.

[5] D. H. Wright, "A simple, stable model of mutualism incorporating handling time," American Naturalist, vol. 134, no. 4, pp. 664-667, 1989.

[6] J. García-Algarra, J. Galeano, J. M. Pastor, J. M. Iriondo, and J. J. Ramasco, "Rethinking the logistic approach for population dynamics of mutualistic interactions," Journal of Theoretical Biology, vol. 363, pp. 332-343, 2014.

[7] C. S. Holling, "Some characteristics of simple types of predation and parasitism," The Canadian Entomologist, vol. 91, no. 7, pp. 385-398, 1959.

[8] S. R.-J. Jang, "Dynamics of herbivore-plant-pollinator models," Journal of Mathematical Biology, vol. 44, no. 2, pp. 129-149, 2002.

[9] T. K. Kar and A. Ghorai, "Dynamic behaviour of a delayed predator-prey model with harvesting," Applied Mathematics and Computation, vol. 217, no. 22, pp. 9085-9104, 2011.

[10] Y. Wang and M. Zhao, "Dynamic analysis of an impulsively controlled predator-prey model with Holling type IV functional response," Discrete Dynamics in Nature and Society, vol. 2012, Article ID 141272, 18 pages, 2012.

[11] P. J. Pal, P. K. Mandal, and K. K. Lahiri, "A delayed ratiodependent predator-prey model of interacting populations with Holling type III functional response," Nonlinear Dynamics, vol. 76, no. 1, pp. 201-220, 2014.

[12] L. Zu, D. Jiang, and D. O’Regan, “Conditions for persistence and ergodicity of a stochastic Lotka-Volterra predator-prey model with regime switching," Communications in Nonlinear Science and Numerical Simulation, vol. 29, no. 1-3, pp. 1-11, 2015.

[13] M. Liu and C. Bai, "Global asymptotic stability of a stochastic delayed predator-prey model with Beddington-DeAngelis functional response," Applied Mathematics and Computation, vol. 226, pp. 581-588, 2014.

[14] J. P. Tripathi, S. Tyagi, and S. Abbas, "Global analysis of a delayed density dependent predator-prey model with Crowley-Martin functional response," Communications in Nonlinear Science and Numerical Simulation, vol. 30, no. 1-3, pp. 45-69, 2016.

[15] M. Ouyang and X. Li, "Permanence and asymptotical behavior of stochastic prey-predator system with Markovian switching," Applied Mathematics and Computation, vol. 266, pp. 539-559, 2015.

[16] A. Mougi and M. Kondoh, "Stability of competition-antagonism-mutualism hybrid community and the role of community network structure," Journal of Theoretical Biology, vol. 360, pp. 54-58, 2014. 
[17] Y. Kuang, Delay Differential Equations: with Applications in Population Dynamics, vol. 191 of Mathematics in Science and Engineering, Academic Press, Boston, Mass, USA, 1993.

[18] X. Mao and C. Yuan, Stochastic Differential Equations with Markovian Switching, Imperial College Press, London, UK, 2006.

[19] D. Jana, R. Agrawal, and R. K. Upadhyay, "Dynamics of generalist predator in a stochastic environment: effect of delayed growth and prey refuge," Applied Mathematics and Computation, vol. 268, pp. 1072-1094, 2015.

[20] L. Zou, Z. Xiong, and Z. Shu, "The dynamics of an ecoepidemic model with distributed time delay and impulsive control strategy," Journal of the Franklin Institute, vol. 348, no. 9, pp. 2332-2349, 2011.

[21] M. Liu and P. S. Mandal, "Dynamical behavior of a one-prey two-predator model with random perturbations," Communications in Nonlinear Science and Numerical Simulation, vol. 28, no. 1-3, pp. 123-137, 2015.

[22] M. Slatkin, "The dynamics of a population in a Markovian environment," Ecology, vol. 59, no. 2, pp. 249-256, 1978.

[23] Q. Luo and X. Mao, "Stochastic population dynamics under regime switching," Journal of Mathematical Analysis and Applications, vol. 334, no. 1, pp. 69-84, 2007.

[24] X. Li, A. Gray, D. Jiang, and X. Mao, "Sufficient and necessary conditions of stochastic permanence and extinction for stochastic logistic populations under regime switching," Journal of Mathematical Analysis and Applications, vol. 376, no. 1, pp. 1128, 2011.

[25] X. Li, X. Mao, and Y. Shen, "Approximate solutions of stochastic differential delay equations with Markovian switching," Journal of Difference Equations and Applications, vol. 16, no. 2-3, pp. 195207, 2010.

[26] P. E. Kloeden and T. Shardlow, "The Milstein scheme for stochastic delay differential equations without using anticipative calculus," Stochastic Analysis and Applications, vol. 30, no. 2, pp. 181-202, 2012.

[27] M. Liu, K. Wang, and Q. Wu, "Survival analysis of stochastic competitive models in a polluted environment and stochastic competitive exclusion principle," Bulletin of Mathematical Biology, vol. 73, no. 9, pp. 1969-2012, 2011.

[28] S. J. Schreiber, "Persistence for stochastic difference equations: a mini-review," Journal of Difference Equations and Applications, vol. 18, no. 8, pp. 1381-1403, 2012.

[29] I. Vrkoč and V. Křivan, "Asymptotic stability of tri-trophic food chains sharing a common resource," Mathematical Biosciences, vol. 270, pp. 90-94, 2015.

[30] Y. Hu, M. Yan, and Z. Xiang, "An impulsively controlled threespecies prey-predator model with stage structure and birth pulse for predator," Discrete Dynamics in Nature and Society, vol. 2015, Article ID 380492, 13 pages, 2015.

[31] M. Liu, C. Bai, M. Deng, and B. Du, "Analysis of stochastic two-prey one-predator model with Lévy jumps," Physica A: Statistical Mechanics and Its Applications, vol. 445, pp. 176-188, 2016.

[32] Q. Liu, "Asymptotic properties of a stochastic $n$-species GilpinAyala competitive model with Lévy jumps and Markovian switching," Communications in Nonlinear Science and Numerical Simulation, vol. 26, no. 1-3, pp. 1-10, 2015.

[33] C. Chen and Y. Kang, "Dynamics of a stochastic multi-strain SIS epidemic model driven by Lévy noise," Communications in Nonlinear Science and Numerical Simulation, vol. 42, pp. 379395, 2017. 


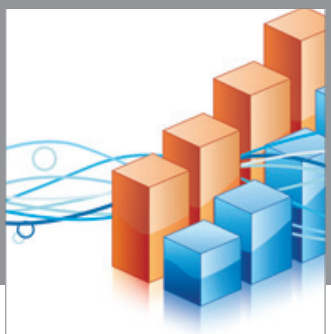

Advances in

Operations Research

vatem alat4

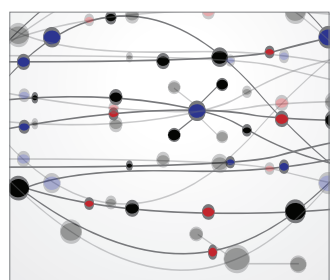

\section{The Scientific} World Journal
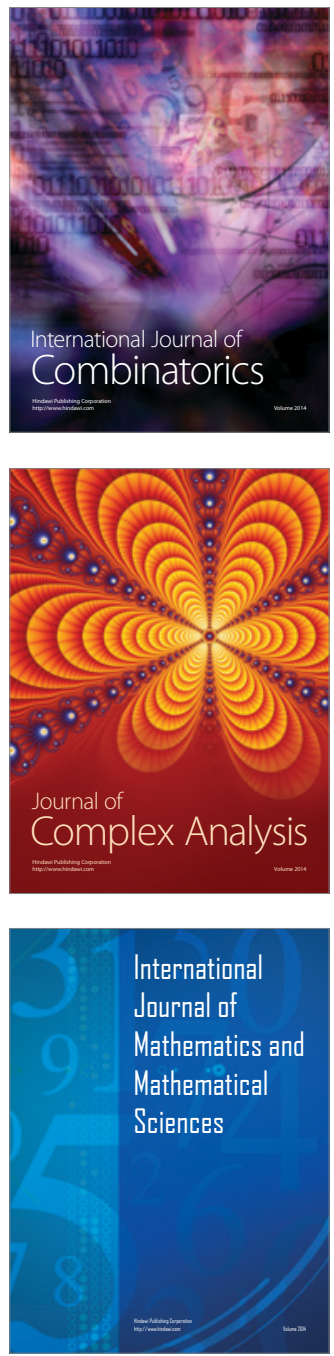
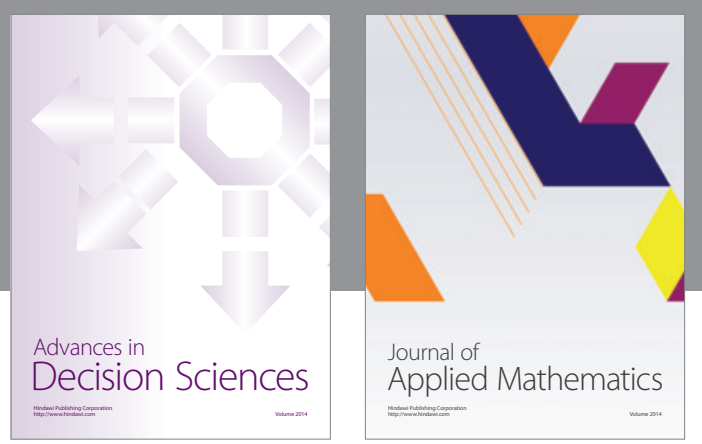

Algebra

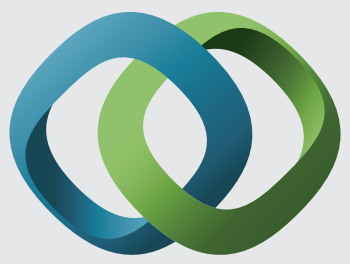

\section{Hindawi}

Submit your manuscripts at

http://www.hindawi.com
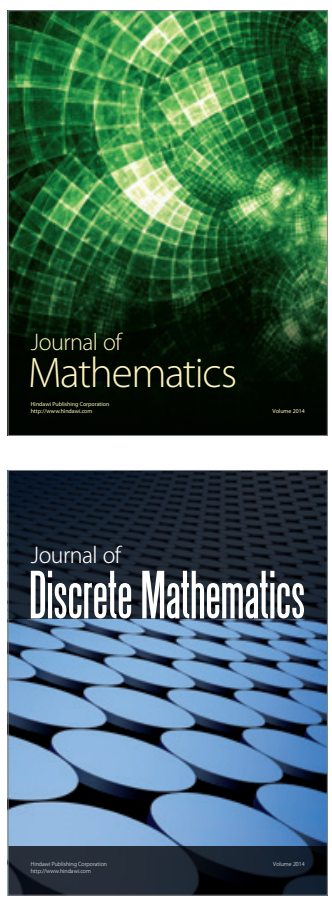

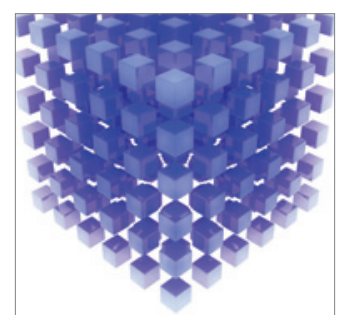

Mathematical Problems in Engineering
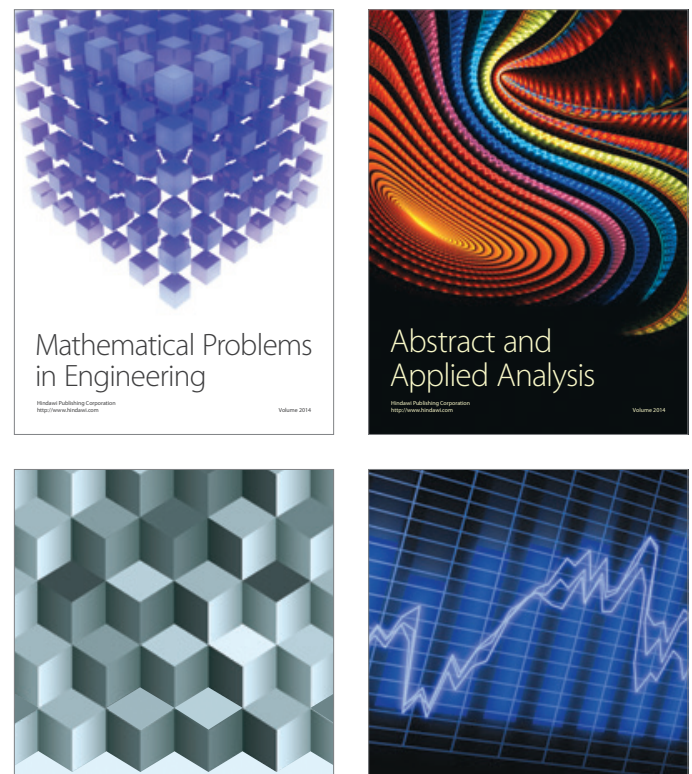

Journal of

Function Spaces

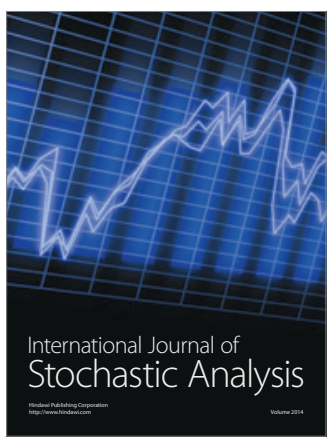

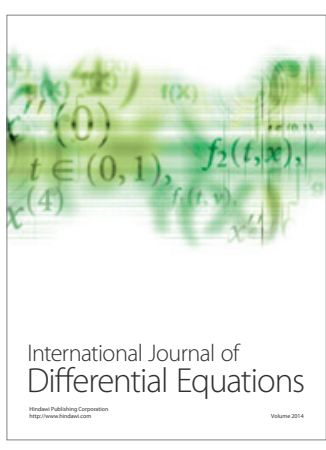
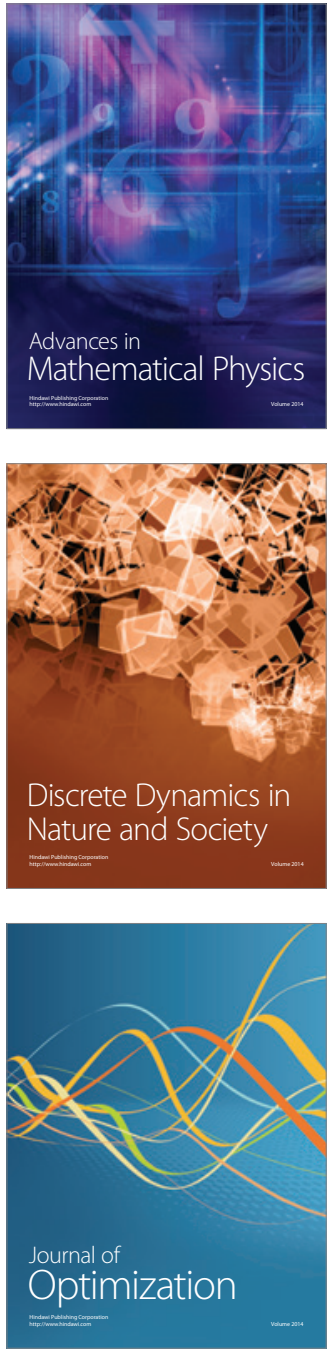